QUARTERLY OF APPLIED MATHEMATICS

VOLUME LXX, NUMBER 4

DECEMBER 2012, PAGES 625-657

S $0033-569 X(2012) 01253-2$

Article electronically published on June 21, 2012

\title{
THE VALUE FUNCTION OF THE SHALLOW LAKE PROBLEM AS A VISCOSITY SOLUTION OF A HJB EQUATION
}

\author{
BY \\ GEORGIOS KOSSIORIS (Department of Mathematics, University of Crete, Knossou Avenue, P.O. \\ BOX 2208, Heraklion 71409, Greece) \\ AND \\ CHRISTOS ZOHIOS (Department of Mathematics, University of Crete, Knossou Avenue, P.O. BOX \\ 2208, Heraklion 71409, Greece)
}

\begin{abstract}
The economic analysis of a shallow lake ecological system requires the study of a nonstandard optimal control problem due to the conflicting services it provides and the nonlinearity of the governing dynamics. We first investigate the geometry of the optimal control-optimal path pair, by standard control analysis, for a given range of the discount factor. We then consider the welfare function (value function) as a viscosity solution of a reduced Hamilton-Jacobi-Bellman equation and we prove various regularity properties which are related to the dynamics of the problem. Finally, we approximate the welfare function by monotone convergent numerical schemes and present the numerical results.
\end{abstract}

1. Introduction-description of the physical problem. The purpose of this work is the theoretical and numerical study of the welfare function describing the economics of shallow lakes, as a viscosity solution of a Hamilton-Jacobi-Bellman equation. The pollution of shallow lakes is a phenomenon which is quite often observed, due to the heavy use of fertilizers on the surrounding land and an increased inflow of waste water from human settlements and industries. The shallow lake system provides conflicting services as a resource, due e.g. to the provision of ecological services of a clear lake, and as a waste sink, due e.g. to agricultural activities. The economic analysis of the system requires the study of an optimal control problem, or a differential game in the case of common property resources by various communities; see e.g. [8], [5] and [15.

Received September 20, 2010.

2010 Mathematics Subject Classification. Primary 49L25.

The authors would like to thank Prof. A. Xepapadeas and Prof. G. Zouraris for stimulating discussions and suggestions.

E-mail address: kosioris@math.uoc.gr

E-mail address: zohios@math.uoc.gr

(C) 2012 Brown University 
The dynamics of the problem are described by

$$
\left\{\begin{array}{l}
y^{\prime}(t)=\alpha(t)-b \cdot y(t)+\frac{y^{2}(t)}{y^{2}(t)+1}, \quad t>0 \\
y(0)=x_{0}
\end{array}\right.
$$

where $\alpha(t)=\sum_{i=1}^{n} \alpha_{i}(t)$ and $\alpha_{i}$ is the loading of phosphorus that every community loads in the lake, $y(t)$ is the amount of phosphorus in algae and $b$ is the rate of loss, consisting of sedimentation, outflow and sequestration in other biomass. The last term on the right-hand side is the rate of recycling of phosphorus. Sediment resuspension, due e.g. to waves, or oxygen depletion might be the causes of recycling. In this model the overall recycling rate is assumed to be a sigmoid function; see [8].

The lake has value as a waste sink for agriculture $\left(\ln \left(\alpha_{i}\right)\right)$ and it provides ecological services that decrease with the amount of phosphorus $\left(-c y^{2}\right)$. So, the running cost for the $i$-th community is $\ln \left(\alpha_{i}\right)-c y^{2}$. We assume that $\alpha_{i}(t)>0, t \in[0,+\infty)$. The parameter $c$ reflects the relative weight of these welfare components (large $c$ gives more weight to the ecological services of the lake). Assuming that we are over an infinite horizon, the objectives become

$$
W_{i}=\int_{0}^{\infty} e^{-\rho t}\left[\ln \left(\alpha_{i}(t)\right)-c y_{x_{0}}^{2}(t, \alpha)\right] d t, \quad i=1, \ldots, n,
$$

where $\rho>0$ is the discount factor. Optimal management of the lake requires maximizing the sum of the objectives $W_{i}$,

$$
W\left(x_{0}\right)=\sup _{\left(\alpha_{1}, \ldots, \alpha_{n}\right) \in A} \int_{0}^{\infty} e^{-\rho t}\left[\sum_{i=1}^{n} \ln \left(\alpha_{i}(t)\right)-n c y_{x_{0}}^{2}(t, \alpha)\right] d t,
$$

subject to the dynamics (1.1); see [15]. Here, $a=\sum_{i=1}^{n} a_{i}, A$ is the set of all measurable functions $\left(\alpha_{1}, \ldots, \alpha_{n}\right):[0,+\infty) \rightarrow B$ and $B \subseteq \mathbb{R}^{n}$. This is an optimal control problem and according to the classical optimal control theory the function $W(\cdot)$ is expected to satisfy the equation

$$
\rho W(x)=H(x, D W),
$$

where the Hamiltonian $H$ is given by

$$
H(x, \lambda)=\sup _{\left(a_{1}, \ldots, a_{n}\right) \in B}\left\{\left(a-b x+\frac{x^{2}}{x^{2}+1}\right) \lambda+\sum_{i=1}^{n} \ln \left(a_{i}\right)-n c x^{2}\right\} .
$$

This is the fundamental idea of dynamic programming according to which $W$ satisfies a functional equation often called the Dynamic Programming Principle, and when $W$ is smooth enough, its infinitesimal version yields the above (HJB) equation; see e.g. [11, [16. In general, $W$ is not expected to be differentiable. This is the case for various values of the discount factor, where a vortex in the optimal control-space phase diagram appears. A Skiba point is present and the optimal path jumps from an upper trajectory to a lower one, or the opposite; see [18], [15], 21]. As a result, the welfare function is expected to undergo a change of slope and this makes $W$ nonsmooth at the Skiba point. Thus, equation (HJB) should be considered in a generalized sense. The nonsmooth welfare (value) function (1.3) is expected to be continuous and bounded. Viscosity solutions, first introduced by Crandall and Lions [10, (see also the review article [9]) provide the 
right framework to study (HJB). An overview of the results in deterministic optimal control theory within the framework of viscosity solutions is presented in 22] see also 3 .

In the present work we first study our control problem on a compact control space and we prove monotonicity properties for the corresponding value function as a viscosity solution of a modified Hamilton-Jacobi equation. These properties are inherited in the limit to the value function (1.3). We exploit these properties to show that (HJB) reduces to the following Hamilton-Jacobi equation:

$$
\rho W(x)-\left(\frac{x^{2}}{x^{2}+1}-b x\right) D W+2\left(\ln (-D W)+x^{2}+1\right)=0 .
$$

We further study the regularity of (1.3) as the viscosity solution of (OHJB) and relate it with the dynamics of the optimal control problem. We finally approximate (1.3) by means of monotone convergent schemes and present various numerical results. A simpler problem was studied in [12.

The paper is organized as follows. In Section 2 we study the optimal dynamics of the problem given by the Pontryagin Maximum Principle for all values of the discount factor $\rho$ in $(0,0.4]$ and investigate the number and the form of the steady-states. In Section 3 we show that we can consider the value function of the shallow lake problem as a viscosity solution of (OHJB). We also study the regularity of the value function. In Section 4 we derive monotone numerical schemes approximating the viscosity solution, based on finite difference space discretizations. The convergence of these schemes is also proved in this section. In Section 5 we compare the numerical results produced by these schemes with the results obtained by the Simple Shooting Method.

2. Control problem analysis for different discount factor values. The Pontryagin Maximum Principle is the most classical tool in optimal control theory (see e.g. [16]) yielding a necessary condition of optimality. Under standard concavity assumptions, the resulting Euler's equations along with the proper transversality condition are also known to be sufficient for optimality; see [16. As will also be clear from the subsequent application of the Pontryagin Maximum Principle, in an $n$-agent game, an optimal strategy is equal to an optimal control of the one-agent problem with the parameter $c$ replaced by $c / n$ (see [15] for further details). Let us consider $n=2$ and $c=1$. In our case, the maximum principle states that, if $c(t)=\left(\alpha_{1}(t), \alpha_{2}(t)\right)$ is an optimal control for the initial state $x_{0} \in \mathbb{R}$ and $x(t):=y_{x_{0}}(t, \alpha)$ is the associated optimal path, where $\alpha(t)=\alpha_{1}(t)+\alpha_{2}(t)$, then there exists a continuous and piecewise continuously differentiable function $\tilde{\lambda}(t)$, called the adjoint function or adjoint variable, such that, if

$$
\bar{H}(x, c, \tilde{\lambda}, t)=\left(\alpha-b x+\frac{x^{2}}{x^{2}+1}\right) \tilde{\lambda}+\left[\ln \left(\alpha_{1}\right)+\ln \left(\alpha_{2}\right)-2 x^{2}\right] e^{-\rho t},
$$

then

$$
\max _{\left(u_{1}, u_{2}\right) \in B} \bar{H}\left(x(t),\left(u_{1}, u_{2}\right), \tilde{\lambda}(t), t\right)=\bar{H}(x(t), c(t), \tilde{\lambda}(t), t)
$$

and

$$
\tilde{\lambda}^{\prime}(t)=-\bar{H}_{x}(x(t), c(t), \tilde{\lambda}(t), t)
$$


From (2.1) we deduce that

$$
\frac{\partial \bar{H}}{\partial u_{1}}(x(t), c(t), \tilde{\lambda}(t), t)=\frac{\partial \bar{H}}{\partial u_{2}}(x(t), c(t), \tilde{\lambda}(t), t)=0 .
$$

Hence,

$$
\lambda(t)+\frac{1}{\alpha_{i}(t)}=0, \quad i=1,2,
$$

where $\lambda(t):=\tilde{\lambda}(t) e^{\rho t}$. From (2.2) we have

$$
\lambda^{\prime}(t)=\left((b+\rho)-\frac{2 x(t)}{\left(x^{2}(t)+1\right)^{2}}\right) \lambda(t)+4 x(t) .
$$

By relation (2.3) we deduce that

$$
\alpha_{1}(t)=\alpha_{2}(t)=-\frac{1}{\lambda(t)}=\frac{\alpha(t)}{2} .
$$

Equation (2.4) using (2.5) is written as

$$
\alpha^{\prime}(t)=-\left((b+\rho)-\frac{2 x(t)}{\left(x^{2}(t)+1\right)^{2}}\right) \alpha(t)+2 x(t) \alpha^{2}(t) .
$$

From equations (1.1) and (2.6) we obtain the following O.D.E. autonomous system:

$$
\left\{\begin{array}{l}
x^{\prime}(t)=\alpha(t)-b \cdot x(t)+\frac{x^{2}(t)}{x^{2}(t)+1}, \\
\alpha^{\prime}(t)=-\left((b+\rho)-\frac{2 x(t)}{\left(x^{2}(t)+1\right)^{2}}\right) \alpha(t)+2 x(t) \alpha^{2}(t) .
\end{array}\right.
$$

In the sequel we will denote the right-hand sides of the first and the second equation in (2.7) by $f_{1}(x, \alpha)$ and $f_{2}(x, \alpha)$, respectively.

The dynamics of the system (2.7) have been described in [21], where a bifurcation analysis for arbitrary values of $\rho$ and $b$ is presented. If $\alpha$ is constant the first equation of (2.7) has one steady-state for high values of $\alpha$. For low values of $\alpha$, three different cases can occur away from $(0,0)$ depending on the value of the parameter $b$ : the system can have one steady-state or two steady-states (in this case the third root of the right-hand side of the equation determines the domains of attraction) or low values of $\alpha$ can yield one steady-state again, followed by a range of $\alpha$ 's with two steady states.

For the needs of the present paper, the problem is studied for $\rho \in(0,0.4]$ and $b=0.6$. The discount factor usually takes small positive values, so such a range for $\rho$ is of physical interest. Moreover, for such a value of $b$ we are in the third case mentioned above. We choose this value so that the lake displays hysteresis but a flip from the oligotrophic state to a eutrophic state is reversible (the lake can flip back to an oligotrophic state if $\alpha$ is reduced further than the flip point; see [15]). Let us note that, if we consider an arbitrary value for $b$, all the results produced below would be similar. We will also consider that $x \in[0,2]$ for the rest of the paper.

To be more specific the following lemmas (see the Appendices $\mathrm{A}, \mathrm{B}, \mathrm{C}$ ) describe the dynamics of (2.7):

Lemma 2.1. For $\rho \in(0,0.4]$ and $b=0.6$ the autonomous system (2.7) has only one or three steady-states for $x \in(0,2]$. 


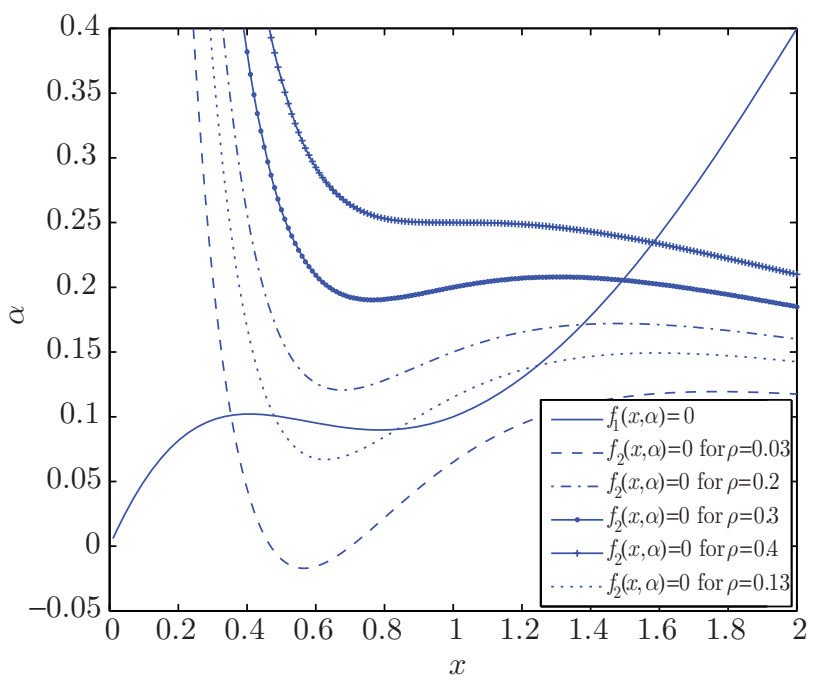

Fig. 1. The $(x, \alpha)$ diagram for different values of $\rho(b=0.6)$. The two cases of one and of three steady-states (which are the intersection points of the curves $f_{1}(x, \alpha)=0$ and $\left.f_{2}(x, \alpha)=0\right)$ are depicted.

Lemma 2.2. In case the system (2.7) has only one steady-state, under the conditions described in Lemma 2.1, this is a saddle point. In case we have three steady-states, the first and the third are saddle-point stable and the point in the middle is a vortex, that is, unstable with a stability matrix with complex eigenvalues (with the exception of degeneracies, which appear when $\rho$ is near the boundary of the range in which we have three steady-states).

In Fig. 1 we can see the steady-states (the intersection points of the curves $f_{1}(x, \alpha)=0$ and $\left.f_{2}(x, \alpha)=0\right)$ for different values of $\rho$. For the computation of the range of $\rho$ in which each of the two cases described in Lemma 2.1 occur, see Appendix B. In the classical theory the candidates for optimality are the stable manifolds (see e.g. [15], 21]).

In the case of one saddle point $\left(x_{s 0}, \alpha_{s 0}\right)$, the stable (and unstable) manifold is shown in Fig. 2, where the curves $f_{1}(x, \alpha)=0$ and $f_{2}(x, \alpha)=0$ are also shown. The dynamics of the system (2.7) along with the transversality condition $\lim _{t \rightarrow \infty} \lambda(t) e^{-\rho t}=0$ make the stable manifolds the only admissible curves for optimality (see [21]).

Given the definition of a saddle point, we can compute the value of the welfare function (1.3) at $x_{s 0}$ by means of

$$
\begin{aligned}
W\left(x_{s 0}\right) & =\max _{\left(\alpha_{1}, \alpha_{2}\right) \in A} \int_{0}^{\infty} e^{-\rho t}\left[\ln \left(\alpha_{1}(t)\right)+\ln \left(\alpha_{2}(t)\right)-2 y_{x_{s 0}}^{2}(t, \alpha)\right] d t \\
& =\int_{0}^{\infty} e^{-\rho t}\left[2 \ln \left(\frac{\alpha_{s 0}}{2}\right)-2 x_{s 0}^{2}\right] d t \\
& =\left(2 \ln \left(\frac{\alpha_{s 0}}{2}\right)-2 x_{s 0}^{2}\right) \int_{0}^{\infty} e^{-\rho t} d t
\end{aligned}
$$




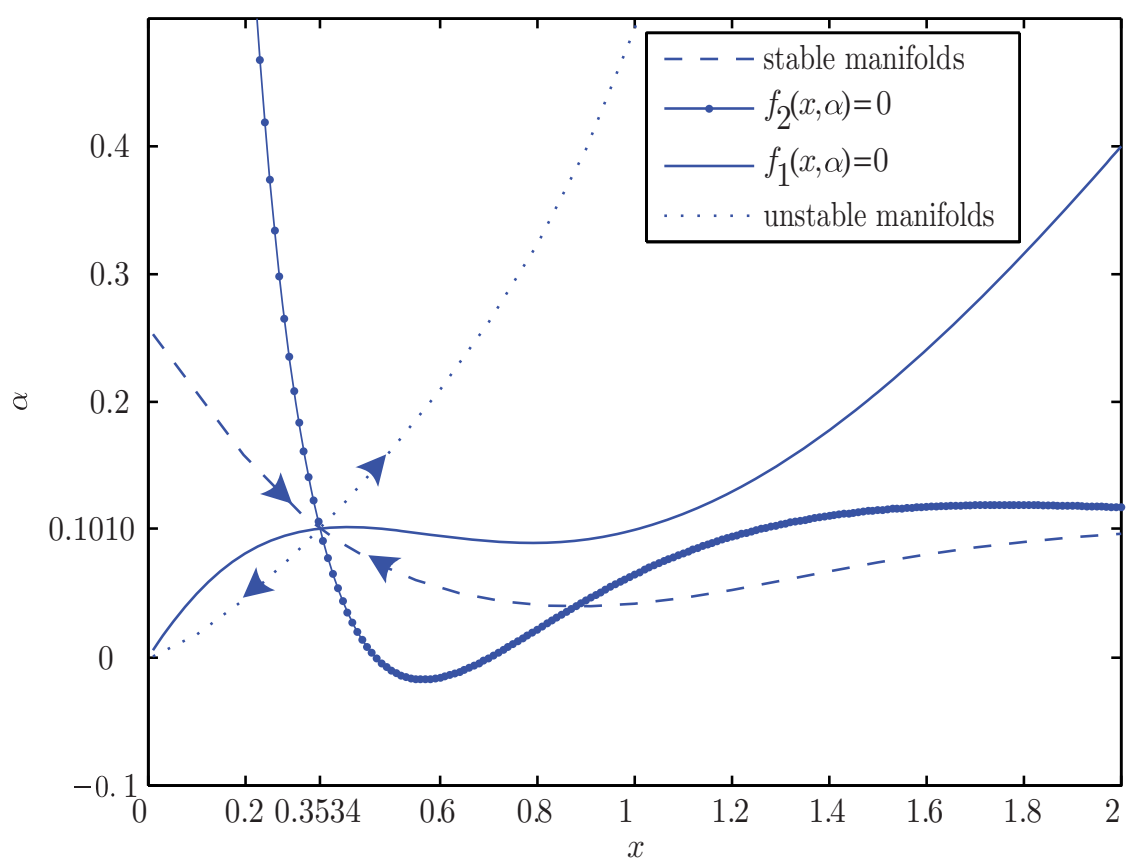

FIG. 2. Example of an $(x, \alpha)$ diagram in the case of one saddle point for $b=0.6$ and $\rho=0.03$.

that is,

$$
W\left(x_{s 0}\right)=\left(2 \ln \left(\frac{\alpha_{s 0}}{2}\right)-2 x_{s 0}^{2}\right) \cdot \frac{1}{\rho} .
$$

The welfare function at any point $x$ can be found via the stable manifold through integration, as follows:

$W(x)=W\left(x_{s 0}\right)+\int_{x_{s 0}}^{x} D W(k) d k=W\left(x_{s 0}\right)+\int_{x_{s 0}}^{x} \lambda(k) d k \stackrel{2.5}{=} W\left(x_{s 0}\right)+\int_{x_{s 0}}^{x}-\frac{2}{\alpha(k)} d k$.

Figure 3 shows the welfare function, in the case of one saddle point, obtained by a numerical integration in (2.9), where the stable manifolds were approximated numerically by a Simple Shooting Method (see Sec. 5).

In the case of two saddle points $x_{s 1}, x_{s 2}$ and a vortex $x_{s 3}$, the stable manifolds are shown in Fig. 4. In this case it can be proved that there is a point $x_{s} \in\left[x_{1}, x_{2}\right]$, where $x_{1}$ and $x_{2}$ are the $x$-coordinates of the intersection points of the outer upper curl and the outer lower curl with the curve representing the steady-states for $x$, respectively, such that

$$
\Delta W\left(x_{s}\right)=0 ; \Delta W(x)<0, x \in\left[x_{1}, x_{s}\right) ; \Delta W(x)>0, x \in\left(x_{s}, x_{2}\right] .
$$

Here, $\Delta W$ is the difference of the welfare functions obtained by integrating the upper trajectory leading to the steady-state on the right $\left(W_{r}\right)$ and the lower trajectory leading 


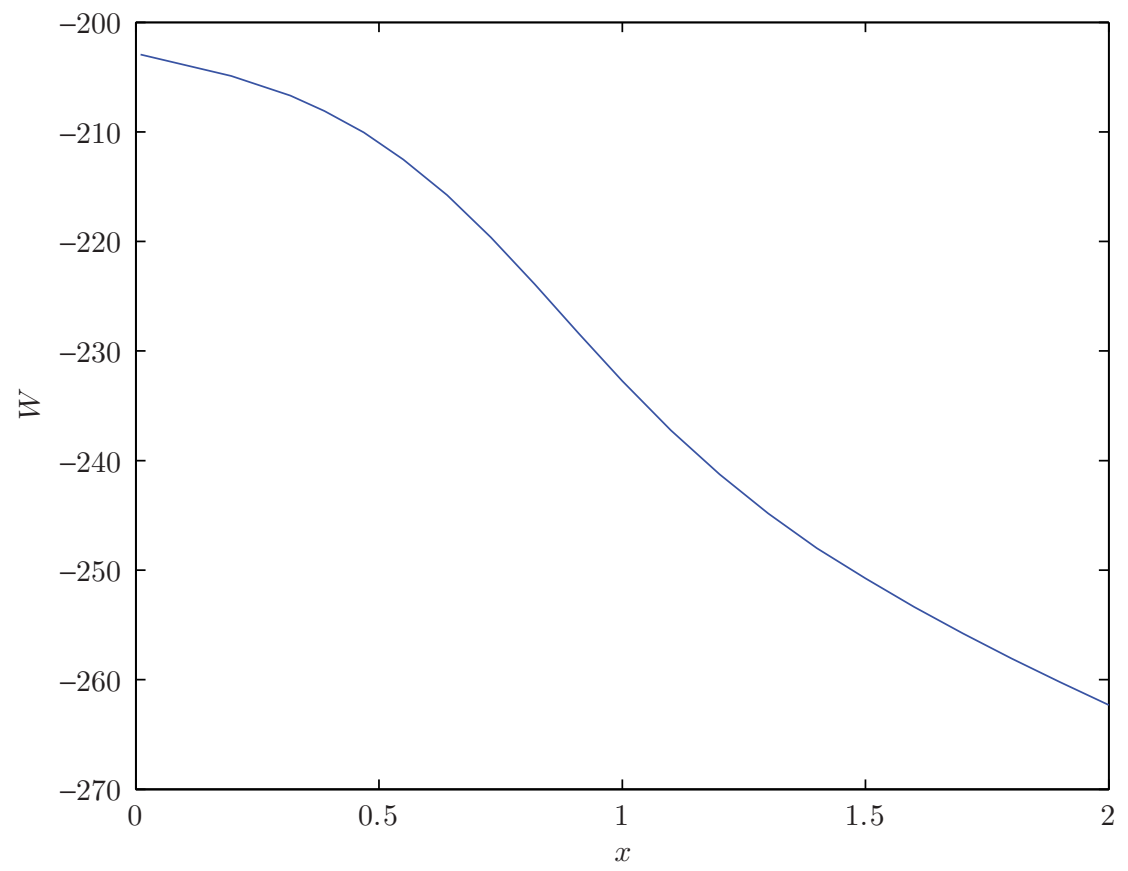

FIG. 3. The welfare function provided by the Simple Shooting Method in the case of one saddle point $(b=0.6, \rho=0.03)$.

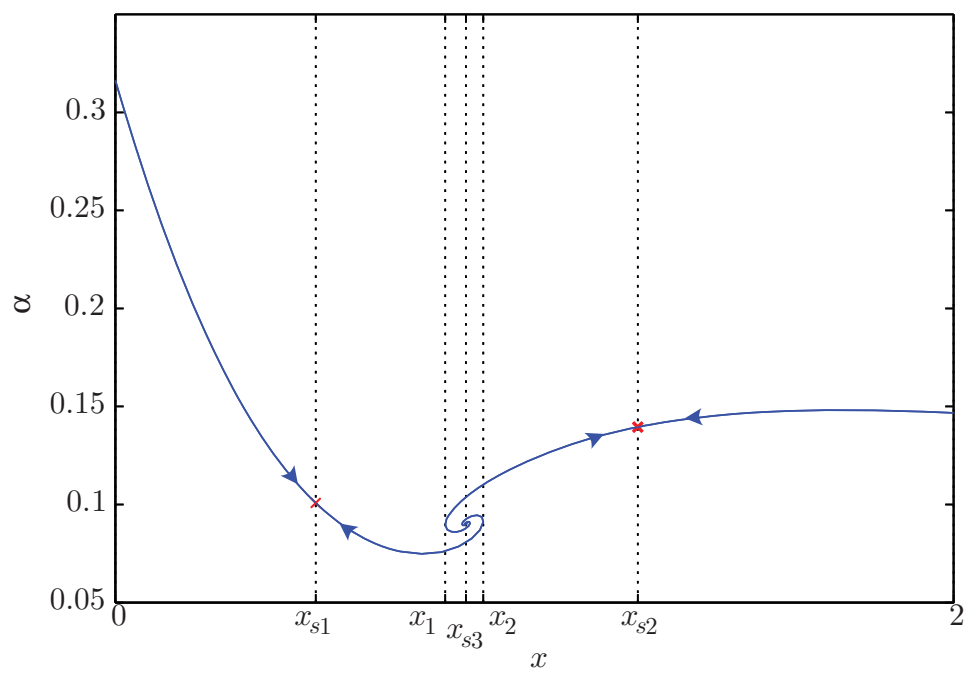

Fig. 4. Example of an $(x, \alpha)$ diagram, where the stable manifolds are shown, in the case of three steady-states. $x_{s 1}$ and $x_{s 2}$ are the two saddle points, $x_{s 3}$ is the vortex and $\left[x_{1}, x_{2}\right]$ is the domain where the set of spirals appears. In this case, $b=0.6$ and $\rho=0.13$. 


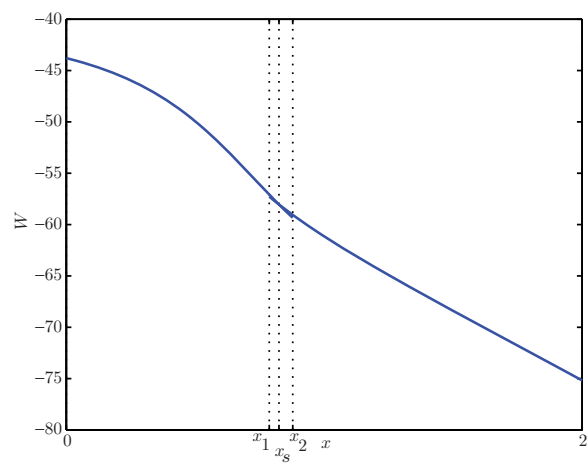

(a)

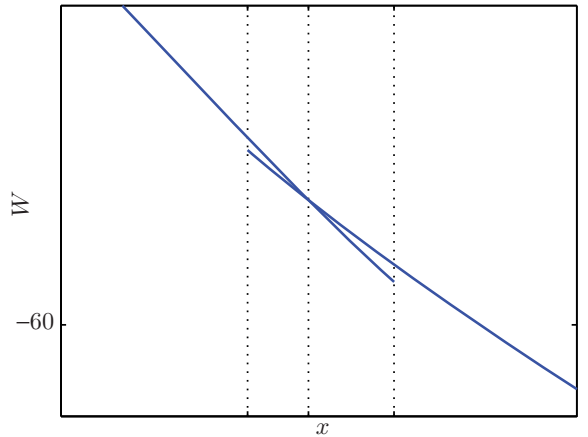

(b)

FIG. 5. The welfare function $W$ provided by the Simple Shooting Method in the case of three steady-states. It is multi-valued in $\left[x_{1}, x_{2}\right]$ and there is an intersection point of $W_{l}$ and $W_{r}$ in this domain, which is the Skiba point $x_{s}$. In (b), Fig. (a) is zoomed around $x_{s}$.

to the steady-state on the left $\left(W_{l}\right)$ by means of equation (2.9). The point $x_{s}$ (see the Appendix of [15] for the proof of the existence of this point) is called a Skiba point and it can be approximated as the intersection point of $W_{r}$ and $W_{l}$ ( $W$ is multi-valued in $\left[x_{1}, x_{2}\right]$; see Fig. [5]). Let us note that a Skiba point exists only in the case that the curve $\left(f_{1}(x, \alpha)=0\right)$ intersects both the outer upper and the outer lower curves (i.e. if $\left[x_{1}, x_{2}\right] \neq \emptyset$ ) and this is not always the case (see [21]). Relation (2.10) implies that if the initial amount of phosphorus $x_{0}$ is on the left-hand side of the Skiba point, the equilibrium jumps to the lower trajectory and moves towards the steady-state on the left and if the initial amount of phosphorus $x_{0}$ is on the right-hand side of the Skiba point, the equilibrium jumps to the upper trajectory and moves towards the steady-state on the right. The optimal trajectory provided by the Simple Shooting Method and the jump of the control at $x_{s}$ are depicted in Fig. 6.

In this work we present an alternative way to study the problem of optimality through the equation (HJB), with boundary conditions (2.8), using the framework of viscosity solutions. This approach is presented in the following section.

\section{Study of the value function (Welfare function).}

3.1. Consideration of the value function as a viscosity solution. Following [10, a continuous function $V$ is a viscosity solution of the equation (HJB) in $O \subseteq \mathbb{R}$, where $O$ is an open interval, if the following conditions are satisfied:

(i) For any $\phi \in C^{1}(O)$, if $y \in O$ is a local maximum point for $V-\phi$, then

$$
\rho V(y) \leq H(y, D \phi(y)) .
$$

(ii) For any $\phi \in C^{1}(O)$, if $y \in O$ is a local minimum point for $V-\phi$, then

$$
\rho V(y) \geq H(y, D \phi(y)) .
$$




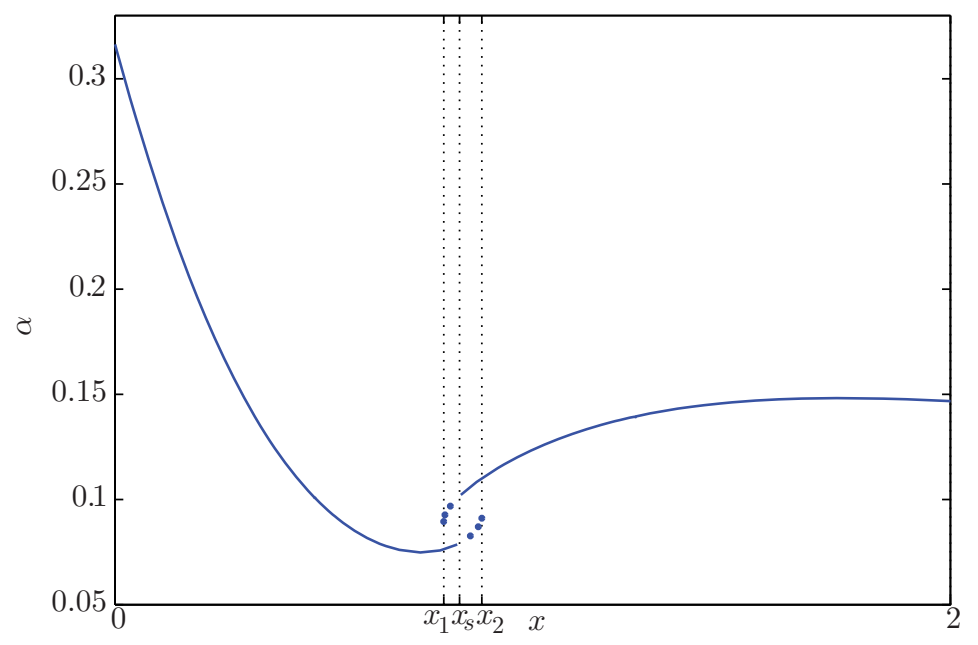

Fig. 6. Due to the existence of a Skiba point $x_{s}$, only the upper trajectory (at the right side of $x_{s}$ ) and the lower trajectory (at the left side of $x_{s}$ ) are kept. The dots show the domain $\left[x_{1}, x_{2}\right]$ in which $\alpha$ is multi-valued.

Any $V$ satisfying (i) is called a viscosity subsolution of the equation (HJB) in $O$, whereas, if (ii) holds, then $V$ is a viscosity supersolution in $O$.

An equivalent way to formulate conditions (i) and (ii), which we will use in the following, is in terms of the superdifferential and the subdifferential of $V$ at a point $x$, denoted by $D^{+} V(x)$ and $D^{-} V(x)$, respectively (for their definition see, for example, 13, page 139). Namely, (i) holds if and only if

(j) $\rho V(y) \leq H(y, \lambda) \quad \forall y \in O, \forall \lambda \in D^{+} V(y)$.

Similarly, (ii) holds if and only if

(jj) $\rho V(y) \geq H(y, \mu) \quad \forall y \in O, \forall \mu \in D^{-} V(y)$.

For the proof of the equivalence of these two definitions, see 2 .

In order to apply the standard theory of viscosity solutions for optimal control, we shall assume that the external loadings $\alpha_{i}, i=1,2$, take values on a bounded interval $(0, M]$. We shall denote the corresponding welfare function (1.3), subsequently called a value function, by $V_{M}$. Our aim is to show that the limiting value function as $M \rightarrow \infty$ is a viscosity solution of the simplified Hamilton-Jacobi-Bellman equation (OHJB) in (0,2).

The value function $V_{M}$ is expected to satisfy the following Hamilton-Jacobi-Bellman equation:

$$
\rho V_{M}(x)-H_{M}\left(x, D V_{M}\right)=0,
$$

where

$$
H_{M}(x, \lambda)=\sup _{\left(a_{1}, a_{2}\right) \in(0, M]^{2}}\left\{\left(a_{1}+a_{2}-b x+\frac{x^{2}}{x^{2}+1}\right) \lambda+\ln \left(a_{1}\right)+\ln \left(a_{2}\right)-2 x^{2}\right\} .
$$

We have the following proposition: 
Proposition 3.1. $V_{M}$ is a viscosity solution of equation (3.1) in $(0,2)$ and this equation admits a Comparison Principle.

Proof. It can easily be proved using calculus that $f(x, c)=a-b x+\frac{x^{2}}{x^{2}+1}$ and $h(x, c)=$ $\ln \left(a_{1}\right)+\ln \left(a_{2}\right)-2 x^{2}$, where $x \in(0,2), c=\left(a_{1}, a_{2}\right) \in(0, M]^{2}$ and $a=a_{1}+a_{2}$, are continuous in $(0,2) \times(0, M]^{2}$, Lipschitz continuous with respect to $x$, uniformly in $c$ and bounded for all $x \in(0,2)$ and $c \in(0, M]^{2}$. Then, using these properties for $f$ and $h$ it is along the lines of standard theory to prove that the problem is well-posed and that $V_{M} \in B U C(0,2)$ (see [2], page 99). From this, using the Dynamic Programming Principle, we deduce that $V_{M}$ satisfies equation (3.1) in the viscosity sense (see [2], page 104). Finally, there is a Comparison Principle for this equation between viscosity suband supersolutions (for its proof, see [2, pages 51-54) which is the following: Let $J$ be a bounded, open subset of $(0,2)$. Assume that $V_{M_{1}}, V_{M_{2}} \in C(\bar{J})$ are, respectively, the viscosity sub- and supersolution of (3.1) in $J$ and $V_{M_{1}} \leq V_{M_{2}}$ on $\partial J$. Then

$$
V_{M_{1}} \leq V_{M_{2}} \text { in } \bar{J} .
$$

REMARK 3.2. The choice of the interval $(0,2]$ is not restrictive, since it captures all the characteristics of the physical problem. The interval can be chosen arbitrarily provided that it includes the critical points of the dynamical system (2.7).

The Hamiltonian $H_{M}$ takes a special form depending on the choice of $\lambda$, as the following lemma shows:

LEMma 3.3. We have that

$$
H_{M}(x, \lambda)=\left\{\begin{array}{lll}
\left(\frac{x^{2}}{x^{2}+1}-b x\right) \lambda-2\left(\ln (-\lambda)+x^{2}+1\right), & \lambda \leq-\frac{1}{M}, & x \in(0,2), \\
\left(2 M-b x+\frac{x^{2}}{x^{2}+1}\right) \lambda+2 \ln (M)-2 x^{2}, & \lambda>-\frac{1}{M}, & x \in(0,2) .
\end{array}\right.
$$

Proof. Let

$$
g\left(a_{1}, a_{2}\right)=\left(a_{1}+a_{2}-b x+\frac{x^{2}}{x^{2}+1}\right) \lambda+\ln \left(a_{1}\right)+\ln \left(a_{2}\right)-2 x^{2}, \quad\left(a_{1}, a_{2}\right) \in \mathbb{R}^{2} .
$$

The point $\left(-\frac{1}{\lambda},-\frac{1}{\lambda}\right)$ is the only critical point of $g$ in $\mathbb{R}^{2}$. We consider the following cases:

- $\lambda \leq-\frac{1}{M}, \quad x \in(0,2)$. Then $-\frac{1}{\lambda} \leq M$, so $\left(-\frac{1}{\lambda},-\frac{1}{\lambda}\right) \in(0, M]^{2}$. Let us assume that there exist $a_{1}, a_{2} \in(0, M]$ such that $g\left(a_{1}, a_{2}\right)>g\left(-\frac{1}{\lambda},-\frac{1}{\lambda}\right)$. Then

$$
\begin{aligned}
& \left(a_{1}+a_{2}-b x+\frac{x^{2}}{x^{2}+1}\right) \lambda+\ln \left(a_{1}\right)+\ln \left(a_{2}\right)-2 x^{2} \\
& >\left(-\frac{2}{\lambda}-b x+\frac{x^{2}}{x^{2}+1}\right) \lambda+\ln \left(-\frac{1}{\lambda}\right)+\ln \left(-\frac{1}{\lambda}\right)-2 x^{2} .
\end{aligned}
$$

Hence,

$$
a_{1} \lambda+\ln \left(-a_{1} \lambda\right)+a_{2} \lambda+\ln \left(-a_{2} \lambda\right)>-2 .
$$

Using the inequality $\ln (x) \leq x-1, \forall x>0$, we get

$$
\ln \left(-a_{1} \lambda\right)+a_{1} \lambda+\ln \left(-a_{2} \lambda\right)+a_{2} \lambda \leq-2,
$$


and due to (3.3) we have a contradiction. We deduce that

$$
g\left(a_{1}, a_{2}\right) \leq g\left(-\frac{1}{\lambda},-\frac{1}{\lambda}\right) \quad \forall\left(a_{1}, a_{2}\right) \in(0, M]^{2}
$$

and $\left(-\frac{1}{\lambda},-\frac{1}{\lambda}\right)$ is a maximum point for $g$ in $(0, M]^{2}$. So,

$$
\begin{aligned}
H_{M}(x, \lambda) & =\sup _{\left(a_{1}, a_{2}\right) \in(0, M]^{2}} g\left(a_{1}, a_{2}\right)=\max _{\left(a_{1}, a_{2}\right) \in(0, M]^{2}} g\left(a_{1}, a_{2}\right)=g\left(-\frac{1}{\lambda},-\frac{1}{\lambda}\right) \\
& =\left(\frac{x^{2}}{x^{2}+1}-b x\right) \lambda-2\left(\ln (-\lambda)+x^{2}+1\right) .
\end{aligned}
$$

- $\lambda \geq 0, \quad x \in(0,2)$. Then, it is obvious that $g$ is increasing as either $a_{1}$ or $a_{2}$ is increasing. This means that the maximum for $g$ in $(0, M]^{2}$ is obtained for $a_{1}=a_{2}=M$, so

$$
H_{M}(x, \lambda)=g(M, M)=\left(2 M-b x+\frac{x^{2}}{x^{2}+1}\right) \lambda+2 \ln (M)-2 x^{2} .
$$

- $0>\lambda>-\frac{1}{M}, \quad x \in(0,2)$. Then $M<-\frac{1}{\lambda}$, so $\left(-\frac{1}{\lambda},-\frac{1}{\lambda}\right) \notin(0, M]^{2}$. We have that

$$
\sup _{\left(a_{1}, a_{2}\right) \in(0, M]^{2}} g\left(a_{1}, a_{2}\right)=\max _{\left(a_{1}, a_{2}\right) \in[\varepsilon, M]^{2}} g\left(a_{1}, a_{2}\right), \quad \varepsilon>0 \text { small, }
$$

because $\lim _{\left(a_{1}, a_{2}\right) \rightarrow(0,0)} g\left(a_{1}, a_{2}\right)=-\infty$. We deduce that the maximum of $g$ in $(\varepsilon, M]^{2}$ will be at the boundary of $[\varepsilon, M]^{2}$. Using simple calculus, we deduce that

$$
\max _{\left(a_{1}, a_{2}\right) \in[\varepsilon, M]^{2}} g\left(a_{1}, a_{2}\right)=g(M, M)=\left(2 M-b x+\frac{x^{2}}{x^{2}+1}\right) \lambda+2 \ln (M)-2 x^{2} .
$$

From (3.4) and (3.5) we deduce that

$$
H_{M}(x, \lambda)=g(M, M)=\left(2 M-b x+\frac{x^{2}}{x^{2}+1}\right) \lambda+2 \ln (M)-2 x^{2} .
$$

Using Proposition 3.1 we have that $V_{M}$, restricted on the domain of interest [0,2], can be considered as a viscosity solution of the following Dirichlet problem:

$$
\left\{\begin{array}{lc}
\rho V_{M}(x)-H_{M}\left(x, D V_{M}\right)=0, & x \in(0,2), \\
V_{M}(0)=v_{0}, \quad V_{M}(2)=v_{1}, & v_{0}, v_{1}<0
\end{array}\right.
$$

where $H_{M}$ is given by Lemma 3.3. Note, however, that the boundary values $v_{0}, v_{1}$ are not in general known and $V_{M}$ can only be computed at the critical points via (2.8).

REMARK 3.4. (i) In the sequel we shall assume that $v_{0}, v_{1}<0$. The negative sign is in accordance with the sign of the welfare function computed at the critical points; see also Figures 3 , 5 .

(ii) Since the trajectories of (1.1) cannot exit from the left boundary of the interval $[0,2]$ and there is always a trajectory that moves strictly out from the right boundary of $[0,2]$ (see [21]), the viscosity solution is expected to be continuous up to the boundary and Dirichlet boundary conditions are satisfied in a classical sense (see [3], 19]).

Some important properties of $V_{M}$, which will be used to prove that the value function is a viscosity solution of the equation (OHJB) in $(0,2)$, are mentioned in the following theorem: 
Theorem 3.5. The viscosity solution $V_{M}$ of the Dirichlet problem (3.6) has the following properties:

(1) The family of functions $\left\{V_{M}\right\}_{M>0}$ is uniformly bounded in [0,2].

(2) $V_{M}$ is locally semiconvex in $(0,2)$ for every $M>0$.

(3) $D^{-} V_{M}(x) \neq \emptyset$ for all $x \in(0,2)$ and $M>0$.

(4) There exists $M_{0} \in \mathbb{R}$ such that for every $M \in \mathbb{R}, M \geq M_{0}$, and for every $x \in(0,2)$ we have that, if $\lambda_{M} \in D^{-} V_{M}(x)$, then $\lambda_{M} \leq-\frac{1}{M}$.

(5) For every $M \geq M_{0}, V_{M}$ is a strictly decreasing function in $(0,2)$.

Proof. 1. Choosing $V_{c}=c \leq v_{0}, v_{1}$ we have:

$$
\begin{aligned}
\rho V_{c}-H_{M}\left(x, D V_{c}\right) & =\rho c-H_{M}(x, 0) \\
& =\rho c-2 \ln (M)+2 x^{2}<0,
\end{aligned}
$$

taking $M$ sufficiently large $(x \in(0,2))$. We deduce that $V_{c}$ is a classical subsolution of (3.6) $\left(V_{c}(0)=c \leq v_{0}, V_{c}(2)=c \leq v_{1}\right)$. By the Comparison Principle we deduce that

$$
c \leq V_{M} \text { in }[0,2] .
$$

We now choose $0<M_{0} \leq M$ and $\tilde{V}=\tilde{u}_{0}-\frac{1}{M_{0}} x$, where $\tilde{u}_{0}$ is a constant (which we will later choose sufficiently large). Since $M_{0} \leq M$, then

$$
\begin{aligned}
\rho \tilde{V}- & H_{M}(x, D \tilde{V})=\rho\left(\tilde{u}_{0}-\frac{1}{M_{0}} x\right)-H_{M}\left(x,-\frac{1}{M_{0}}\right) \\
& =\rho\left(\tilde{u}_{0}-\frac{1}{M_{0}} x\right)-\left(\frac{x^{2}}{x^{2}+1}-b x\right)\left(-\frac{1}{M_{0}}\right)+2 \ln \left(\frac{1}{M_{0}}\right)+2 x^{2}+2 .
\end{aligned}
$$

Terms which depend on $x$ are bounded as $x \in(0,2)$, so, choosing $\tilde{u}_{0}$ sufficiently large we take that $\rho \tilde{V}-H_{M}(x, D \tilde{V})>0$. In addition, $\tilde{u}_{0}$ is taken sufficiently large so that $\tilde{u}_{0}>v_{0}$ and $\tilde{u}_{0}-\frac{2}{M_{0}}>v_{1}$. Then, $\tilde{V}(0)=\tilde{u}_{0}>v_{0}$ and $\tilde{V}(2)=\tilde{u}_{0}-\frac{2}{M_{0}}>v_{1}$. We deduce that $\tilde{V}$ is a classical supersolution of (3.6). By the Comparison Principle, $V_{M} \leq \tilde{V}$ in $[0,2]$; therefore

$$
V_{M} \leq \tilde{u}_{0}-\frac{1}{M_{0}} x \leq \tilde{u}_{0} \quad \text { in }[0,2] .
$$

From (3.7) and (3.8) we have that $c \leq V_{M}(x) \leq \tilde{u}_{0}$, for all $x \in[0,2]$ and $M>0$. Hence, $\left\{V_{M}\right\}_{M>0}$ is uniformly bounded in [0,2].

2. Let $M>0$. It is sufficient to show that

$$
N(x)=-V_{M}(x)=\inf _{\left(\alpha_{1}, \alpha_{2}\right) \in A} \int_{0}^{\infty} e^{-\rho t} h\left(y_{x}(t, c),\left(\alpha_{1}(t), \alpha_{2}(t)\right)\right) d t
$$

is locally semiconcave in $(0,2)$. Let us recall that $f\left(x,\left(a_{1}, a_{2}\right)\right)=a_{1}+a_{2}-b x+\frac{x^{2}}{x^{2}+1}$, $h\left(x,\left(a_{1}, a_{2}\right)\right)=-\left(\ln \left(a_{1}\right)+\ln \left(a_{2}\right)-2 x^{2}\right)$ and $B=(0, M]^{2}$. It can be proved using simple calculus that there is $K_{1}>0\left(K_{1}=b+4\right)$ such that

$$
\left|f\left(x_{2}, c\right)-f\left(x_{1}, c\right)\right| \leq K_{1}\left|x_{2}-x_{1}\right|, \quad \forall x_{1}, x_{2} \in[0,2], \quad \forall c \in(0, M]^{2}
$$

and

$$
\left|f_{x}\left(x_{2}, c\right)-f_{x}\left(x_{1}, c\right)\right| \leq 114\left|x_{2}-x_{1}\right|, \quad \forall x_{1}, x_{2} \in[0,2], \quad \forall c \in(0, M]^{2} .
$$


Moreover, it can easily be proved that

$$
\forall R>0, \quad\left|h\left(x_{2}, c\right)-h\left(x_{1}, c\right)\right| \leq 8\left|x_{2}-x_{1}\right|, \quad \forall x_{1}, x_{2} \in B_{R}, \quad \forall c \in(0, M]^{2} .
$$

In addition, simple calculations yield

$$
\forall R>0, \quad h(x, c)+h(y, c)-2 h\left(\frac{x+y}{2}, c\right) \leq|x-y|^{2}, \quad x, y \in B_{R}, \quad c \in(0, M]^{2} .
$$

The rest of the proof is based on [6] and the proof of Theorem 7.4.11, where a semiconcavity result is obtained for the Bolza problem. Our problem is slightly different (the terminal time is $T=+\infty$ and there is a discount factor $\rho$ ), so, in the following, we adjust the proof to our case. Let $r>0$ be fixed and let $R>0$ be such that all trajectories starting from $B_{r}$ for $t=0\left(x=y_{x}(0, c) \in B_{r}\right)$ stay inside $B_{R}$ in $[0,+\infty)\left(y_{x}(t, c) \in B_{R}\right.$, $t \in[0,+\infty))$. The existence of such an $R$ is proven in Lemma 7.1.2(i) of [6] and the hypotheses of this lemma are satisfied, due to (3.9) and to the fact that the control set $B$ can be set equal to $[\varepsilon, M]^{2}$ for $\varepsilon>0$ small (as there are no optimal controls near 0 ), meaning that $B$ is compact. Given $x, h$ such that $x \pm h \in B_{r}$, let $\tilde{c}:[0,+\infty) \rightarrow(0, M]^{2}$ be an optimal control for $x$. Let us set

$$
y(\cdot)=y_{x}(\cdot, \tilde{c}), \quad y_{-}(\cdot)=y_{x-h}(\cdot, \tilde{c}), \quad y_{+}(\cdot)=y_{x+h}(\cdot, \tilde{c}) .
$$

Using Lemma 7.1.2 ((ii) and (iii)) of [6] (its hypotheses are satisfied due to (3.9), (3.10) and the fact that $B$ is compact, as stated before) we have

$$
\left|y_{+}(s)-y_{-}(s)\right| \leq c|h|, \quad\left|y_{+}(s)+y_{-}(s)-2 y(s)\right| \leq c|h|^{2}
$$

for some constant $c>0$. Using (3.11), (3.12) and (3.13) and following the proof of Theorem 7.4.11 of [6], we have

$$
\begin{aligned}
& h\left(y_{+}(t), \tilde{c}(t)\right)+h\left(y_{-}(t), \tilde{c}(t)\right)-2 h(y(t), \tilde{c}(t)) \\
& \leq c^{2}|h|^{2}+8 c|h|^{2}=C|h|^{2},
\end{aligned}
$$

where $C$ is any constant depending only on $r$. Thus, the definition of $N(x)$, the optimality of $\tilde{c}$ and (3.14) imply that

$$
\begin{aligned}
N(x & +h)+N(x-h)-2 N(x) \\
& \leq \int_{0}^{+\infty} e^{-\rho t}\left[h\left(y_{+}(t), \tilde{c}(t)\right)+h\left(y_{-}(t), \tilde{c}(t)\right)-2 h(y(t), \tilde{c}(t))\right] d t \\
& \leq \int_{0}^{+\infty} e^{-\rho t} \cdot C|h|^{2} d t=\frac{C}{\rho}|h|^{2} .
\end{aligned}
$$

Therefore,

$$
N(x+h)+N(x-h)-2 N(x) \leq C_{1}|h|^{2},
$$

for every $x, h$ such that $x \pm h \in B_{r}$, which proves that $N$ is locally semiconcave in $(0,2)$.

3. Let $M>0$. From 2 we deduce that $V_{M}$ is semiconvex in every compact subset of $(0,2)$. Let $[a, b] \subset(0,2)$. We consider the set

$$
D^{*} V_{M}(x)=\left\{p \in \mathbb{R}: p=\lim _{n \rightarrow+\infty} D V_{M}\left(x_{n}\right), x_{n} \rightarrow x\right\} .
$$


Due to the fact that $V_{M}$ is semiconvex in $[a, b]$, it is a well-known result in nonsmooth analysis (see [1, 7]) that

$$
D^{-} V_{M}(x)=c o D^{*} V_{M}(x), \quad \forall x \in[a, b],
$$

where $c o D^{*} V_{M}(x)$ is the convex hull of $D^{*} V_{M}(x)$. In addition, $V_{M}$ is locally Lipschitz continuous in $(a, b)$ due to its semiconvexity property in $[a, b]$ (see [1, 2] and [6]). By the classical Rademacher theorem, $V_{M}$ is differentiable almost everywhere in $(a, b)$ with locally bounded gradient. From this we deduce that $D^{*} V_{M}(x) \neq \emptyset \forall x \in(a, b)$. Consequently, $D^{-} V_{M}(x) \neq \emptyset \forall x \in(a, b)$, due to (3.15). We conclude that

$$
D^{-} V_{M}(x) \neq \emptyset \quad \forall x \in(0,2) .
$$

4. From 1 we have that there exists $\Lambda>0$ such that

$$
\left|V_{M}(x)\right| \leq \Lambda, \quad \forall x \in(0,2), \quad \forall M>0 .
$$

$V_{M}$ is a viscosity solution of (3.6), so for $M>0, x \in(0,2)$ and $\lambda_{M} \in D^{-} V_{M}(x)$ we deduce using (3.6) and (3.16) that there exists $\Lambda_{1}>0$ such that

$$
H_{M}\left(x, \lambda_{M}\right) \leq \Lambda_{1} .
$$

We now suppose that

$$
\left\{\begin{array}{c}
\forall r \in \mathbb{R} \exists M_{r} \in \mathbb{R}, M_{r} \geq r, \text { and } \exists x_{M_{r}} \in(0,2) \text { and } \lambda_{M_{r}} \in D^{-} V_{M_{r}}\left(x_{M_{r}}\right) \\
\text { such that } \lambda_{M_{r}}>-\frac{1}{M_{r}} .
\end{array}\right.
$$

We have that $a_{r}=-b x_{M_{r}}+\frac{x_{M_{r}}{ }^{2}}{x_{M_{r}}{ }^{2}+1}$ is bounded $\forall r \in \mathbb{R}$, as $x_{M_{r}} \in(0,2)$ for all $r \in \mathbb{R}$. This means that we can choose $r_{0}$ sufficiently large so that, due to (3.18), $M_{r_{0}}$ is sufficiently large to obtain that $2 M_{r_{0}}-b x_{M_{r_{0}}}+\frac{x_{M_{r_{0}}}{ }^{2}}{x_{M_{r_{0}}}+1}>0$. So, from (3.18) we deduce that

$$
2 M_{r}-b x_{M_{r}}+\frac{x_{M_{r}}{ }^{2}}{x_{M_{r}}{ }^{2}+1}>0, \forall r \geq r_{0} .
$$

For $r \geq r_{0}$ we now have, using Lemma 3.3 .

$$
\begin{aligned}
& H_{M_{r}}\left(x_{M_{r}}, \lambda_{M_{r}}\right) \\
& \stackrel{\sqrt{3.18}}{=}\left(2 M_{r}-b x_{M_{r}}+\frac{x_{M_{r}}{ }^{2}}{x_{M_{r}}{ }^{2}+1}\right) \lambda_{M_{r}}+2 \ln \left(M_{r}\right)-2 x_{M_{r}}{ }^{2} \\
& \underset{\geq 3.19}{\geq 3.18}\left(2 M_{r}-b x_{M_{r}}+\frac{x_{M_{r}}{ }^{2}}{x_{M_{r}}{ }^{2}+1}\right)\left(-\frac{1}{M_{r}}\right)+2 \ln \left(M_{r}\right)-2 x_{M_{r}}{ }^{2} \\
& =\quad-2+\frac{1}{M_{r}}\left(b x_{M_{r}}-\frac{x_{M_{r}}{ }^{2}}{x_{M_{r}}{ }^{2}+1}\right)+2 \ln \left(M_{r}\right)-2 x_{M_{r}}{ }^{2} \\
& =\quad-2+\frac{b x_{M_{r}}}{M_{r}}-\frac{1}{M_{r}} \frac{x_{M_{r}}{ }^{2}}{x_{M_{r}}{ }^{2}+1}+2 \ln \left(M_{r}\right)-2 x_{M_{r}}{ }^{2} \\
& \geq \quad-2-\frac{1}{M_{r}}+2 \ln \left(M_{r}\right)-8 .
\end{aligned}
$$

Since by (3.18)

$$
\lim _{r \rightarrow+\infty} M_{r}=+\infty
$$


by taking $r \rightarrow+\infty$ in (3.20) we have that

$$
\lim _{r \rightarrow+\infty} H_{M_{r}}\left(x_{M_{r}}, \lambda_{M_{r}}\right)=+\infty
$$

which contradicts (3.17). So the statement is proved.

5. Let $M \geq M_{0}$. From 3 we deduce that for $x \in(0,2)$ and $\lambda_{M} \in D^{-} V_{M}(x)$ we have that $\lambda_{M} \leq-\frac{1}{M}<0$. So,

$$
\lambda_{M}<0, \quad \forall x \in(0,2), \quad \forall \lambda_{M} \in D^{-} V_{M}(x) .
$$

Let $x_{0} \in(0,2)$. For $x<x_{0}$, using the definition of subdifferential, we have that for every $\lambda_{M_{0}} \in D^{-} V_{M}\left(x_{0}\right)$,

$$
V_{M}(x) \geq V_{M}\left(x_{0}\right)+\lambda_{M_{0}}\left(x-x_{0}\right)+o\left(\left|x-x_{0}\right|\right) .
$$

Therefore,

$$
\limsup _{x \rightarrow x_{0}^{-}} \frac{V_{M}(x)-V_{M}\left(x_{0}\right)}{x-x_{0}} \leq \lambda_{M_{0}} .
$$

Using (3.21) we deduce that $\lim \sup _{x \rightarrow x_{0}^{-}} \frac{V_{M}(x)-V_{M}\left(x_{0}\right)}{x-x_{0}}<0$. This means that there exists $\varepsilon>0$ such that, for every $\delta \in(0, \varepsilon), \frac{V_{M}(x)-V_{M}\left(x_{0}\right)}{x-x_{0}}<0$ holds for $x \in\left(x_{0}-\delta, x_{0}\right)$. So we have that there exists $\delta>0$ such that $V_{M}(x)>V_{M}\left(x_{0}\right), \forall x \in\left(x_{0}-\delta, x_{0}\right)$. The choice of $x_{0} \in(0,2)$ was arbitrary and we deduce that

$$
\forall x_{0} \in(0,2) \exists \delta>0 \text { such that } V_{M}(x)>V_{M}\left(x_{0}\right), \forall x \in\left(x_{0}-\delta, x_{0}\right) .
$$

Let us now assume that $V_{M}$ is not a strictly decreasing function on $(0,2)$. So, there exist $x_{1}, x_{2} \in(0,2), x_{1}<x_{2}$, such that $V_{M}\left(x_{1}\right) \leq V_{M}\left(x_{2}\right)$. According to (3.22), $\exists \delta^{\prime}>0$ such that $V_{M}(x)>V_{M}\left(x_{2}\right), \forall x \in\left(x_{2}-\delta^{\prime}, x_{2}\right)$. This means that there exists $x_{3} \in\left(x_{2}-\delta^{\prime}, x_{2}\right)$ such that $V_{M}\left(x_{3}\right)>V_{M}\left(x_{2}\right)$. Obviously $x_{1} \notin\left(x_{2}-\delta^{\prime}, x_{2}\right)$, so $x_{1}<x_{2}-\delta^{\prime}<x_{3}$. In addition we have

$$
V_{M}\left(x_{1}\right)<V_{M}\left(x_{3}\right) .
$$

$V_{M}$ is continuous at $\left[x_{1}, x_{3}\right]$, so there exists $x_{m} \in\left[x_{1}, x_{3}\right]$ such that $V_{M}\left(x_{m}\right) \geq V_{M}(x)$, $\forall x \in\left[x_{1}, x_{3}\right]$. From (3.23) we see that $x_{m} \neq x_{1}$, so $x_{m} \in\left(x_{1}, x_{3}\right]$. This means that there exists $\theta>0$ such that $\left(x_{m}-\theta, x_{m}\right) \subseteq\left(x_{1}, x_{3}\right]$. From this we deduce that

$$
V_{M}\left(x_{m}\right) \geq V_{M}(x), \forall x \in\left(x_{m}-\theta, x_{m}\right) .
$$

Applying relation (3.22) for $x_{m}$, we have that there exists $\sigma>0$ such that $V_{M}(x)>$ $V_{M}\left(x_{m}\right), \forall x \in\left(x_{m}-\sigma, x_{m}\right)$. Due to (3.24) we come to a contradiction. So we conclude that $V_{M}$ is a strictly decreasing function on $(0,2)$.

The main result of this section is presented in the following proposition:

Proposition 3.6. The value function $V_{M}$ is a viscosity solution of

$$
\left\{\begin{array}{l}
\rho V_{M}(x)-\left(\frac{x^{2}}{x^{2}+1}-b x\right) D V_{M}+2\left(\ln \left(-D V_{M}\right)+x^{2}+1\right)=0 \quad \text { in }(0,2), \\
V_{M}(0)=v_{0}, \quad V_{M}(2)=v_{1}
\end{array}\right.
$$

for all $M \geq M_{0}>0$ and there is at most one continuous solution which will be denoted by $V$. 
Proof. We will first prove that, $\forall M \geq M_{0}, M \in \mathbb{R}^{+}, V_{M}$ is a viscosity solution of

$$
\left\{\begin{array}{l}
\rho V_{M}(x)-\left(\frac{x^{2}}{x^{2}+1}-b x\right) D V_{M}+2\left(\ln \left(-D V_{M}\right)+x^{2}+1\right)=0 \quad \text { in }(0,2), \\
V_{M}(0)=v_{0}, \quad V_{M}(2)=v_{1} .
\end{array}\right.
$$

Let $x \in(0,2)$. Then, from (3) of Theorem 3.5 we have that $D^{-} V_{M}(x) \neq \emptyset$. Let $\lambda_{M} \in D^{-} V_{M}(x)$. Then, from (4) of Theorem 3.5 we get that

$$
\lambda_{M} \leq-\frac{1}{M}
$$

We have the following cases:

- $D^{+} V_{M}(x) \neq \emptyset$. Then $D^{+} V_{M}(x)=D^{-} V_{M}(x)=\left\{D V_{M}(x)\right\}=\left\{\lambda_{M}\right\}$. So, $V_{M}$ is differentiable at $x$ and $D V_{M}(x)=\lambda_{M} \leq-\frac{1}{M}$ from (3.26). From Proposition 3.1 and using Lemma 3.3 we conclude that $V_{M}$ is a viscosity solution of (3.25) at $x$.

- $D^{+} V_{M}(x)=\emptyset$. Then it is explicit that $V_{M}$ is a viscosity subsolution of (3.25) at $x$. From Proposition 3.1 we have that $V_{M}$ is a viscosity supersolution of equation $\rho V_{M}(x)-H_{M}\left(x, D V_{M}\right)=0$ in $(0,2)$. So, using condition (jj) we have that $\rho V_{M}(x)-H_{M}\left(x, \lambda_{M}\right) \geq 0$. Using Lemma 3.3 and (3.26) we get that

$$
\rho V_{M}(x)-\left(\frac{x^{2}}{x^{2}+1}-b x\right) \lambda_{M}-2\left(\ln \left(-\lambda_{M}\right)+x^{2}+1\right) \geq 0 .
$$

The choice of $\lambda_{M} \in D^{-} V_{M}(x)$ is arbitrary, so from (3.27) we deduce that $V_{M}$ is a viscosity supersolution of (3.25) at $x$. We conclude that $V_{M}$ is a viscosity solution of (3.25) at $x$.

These two cases cover all $x \in(0,2)$, so $V_{M}$ is a viscosity solution of (3.25) at $(0,2)$.

The next step is to show that (OHJB) has a unique viscosity solution in $(0,2)$. For this, it is sufficient to prove a comparison theorem for

$$
\left\{\begin{array}{l}
\rho V(x)+H(x, D V)=0 \quad \text { in }(0,2) \\
V(0)=v_{0}, \quad V(2)=v_{1}
\end{array}\right.
$$

where

$$
H(x, \lambda)=-\left(\frac{x^{2}}{x^{2}+1}-b x\right) \lambda+2\left(\ln (-\lambda)+x^{2}+1\right) .
$$

From Theorem 3.1 and Remark 3.3 of Section II of [2] we have that it is sufficient to show that

$$
|H(x, p)-H(y, p)| \leq \omega_{1}(|x-y|(1+|p|)),
$$

for $x, y \in(0,2), p \in \mathbb{R}^{-}$, where $\omega_{1}:[0,+\infty) \rightarrow[0,+\infty)$ is continuous, nondecreasing, with $\omega_{1}(0)=0$. 
Let $x, y \in(0,2), p \in \mathbb{R}^{-}$. Then we have

$$
\begin{aligned}
\mid H(x, & p)-H(y, p)|=| p\left(\frac{y^{2}}{y^{2}+1}-b y-\frac{x^{2}}{x^{2}+1}+b x\right)+2\left(x^{2}-y^{2}\right) \mid \\
& \leq|p| b|x-y|+|p|\left|\frac{y^{2} x^{2}+y^{2}-x^{2} y^{2}-x^{2}}{\left(y^{2}+1\right)\left(x^{2}+1\right)}\right|+2|(x-y)(x+y)| \\
& =|p| b|x-y|+|p|\left|\frac{(y-x)(y+x)}{\left(y^{2}+1\right)\left(x^{2}+1\right)}\right|+2|x-y|(x+y) \\
& =|p| b|x-y|+|p| \frac{|y-x|(y+x)}{\left(y^{2}+1\right)\left(x^{2}+1\right)}+2|x-y|(x+y) \\
& \leq|p| b|x-y|+|p||y-x|(y+x)+2|x-y|(x+y) \\
& \leq|p| b|x-y|+4|p||y-x|+8|x-y| \\
& =8(b+1)|x-y|(|p|+1) .
\end{aligned}
$$

For $\omega_{1}:[0,+\infty) \rightarrow[0,+\infty), \omega_{1}(x)=8(b+1) x$, we have that $\omega_{1}$ is continuous, nondecreasing and $\omega_{1}(0)=0$. Due to (3.31) we get that

$$
|H(x, p)-H(y, p)| \leq \omega_{1}(|x-y|(1+|p|)), \quad \forall x, y \in(0,2), \quad \forall p \in \mathbb{R}^{-}
$$

and the uniqueness of the viscosity solution of the (OHJB) in $(0,2)$ is proven.

We conclude that, if $V$ is the unique continuous viscosity solution of the (OHJB) in $(0,2)$ satisfying the boundary conditions, then

$$
V_{M} \equiv V \quad \forall M \geq M_{0}
$$

REMARK 3.7. The same conclusion can also be drawn for an arbitrary domain $(0, d)$ instead of $(0,2)$, as the proof does not depend on the boundary point of this domain.

The next proposition refers to the smoothness of $V$ based on the results of [13].

Proposition 3.8. (1) The value function $V$ is Lipschitz continuous in $(0,2)$.

(2) In addition, for every $x_{0} \in(0,2), V$ is either differentiable or it has left and right derivatives $V_{x}^{-}\left(x_{0}\right)$ and $V_{x}^{+}\left(x_{0}\right)$ at $x_{0}$, respectively. For $H$ given in (3.29) and $V_{x}^{+}\left(x_{0}\right) \neq V_{x}^{-}\left(x_{0}\right)$, we have

$$
\left\{\begin{array}{l}
H\left(x_{0}, V_{x}^{-}\left(x_{0}\right)\right)=H\left(x_{0}, V_{x}^{+}\left(x_{0}\right)\right)=-\rho W\left(x_{0}\right) \quad \text { and } \\
H\left(x_{0}, l\right)\left(V_{x}^{+}\left(x_{0}\right)-V_{x}^{-}\left(x_{0}\right)\right) \geq-\rho V\left(x_{0}\right)\left(V_{x}^{+}\left(x_{0}\right)-V_{x}^{-}\left(x_{0}\right)\right) \\
\text { for every } l \in\left[\min \left(V_{x}^{-}\left(x_{0}\right), V_{x}^{+}\left(x_{0}\right)\right), \max \left(V_{x}^{-}\left(x_{0}\right), V_{x}^{+}\left(x_{0}\right)\right)\right] .
\end{array}\right.
$$

(3) For every $x_{0} \in(0,2)$ there exists an $a=a\left(x_{0}\right)>0$ such that:

(a) If $-\rho V\left(x_{0}\right)$ is not a local extremum value of $H$, then $\left.V\right|_{\left(x_{0}-a, x_{0}\right]} \in$ $C^{1}\left(\left(x_{0}-a, x_{0}\right]\right)$ and $\left.V\right|_{\left[x_{0}, x_{0}+a\right)} \in C^{1}\left(\left[x_{0}, x_{0}+a\right)\right)$.

(b) If $-\rho V\left(x_{0}\right)$ is a local extremum value of $H$, then the following hold on $\left(x_{0}-a, x_{0}\right]$ (respectively $\left.\left[x_{0}, x_{0}+a\right)\right)$. Either $\left.V\right|_{\left(x_{0}-a, x_{0}\right]} \in C^{1}\left(\left(x_{0}-a, x_{0}\right]\right)$ (respectively $\left.\left.V\right|_{\left[x_{0}, x_{0}+a\right)} \in C^{1}\left(\left[x_{0}, x_{0}+a\right)\right)\right)$ or $V_{x}(x)$ exists for every $\left(x_{0}-\right.$ $\left.a, x_{0}\right]$ (respectively $\left[x_{0}, x_{0}+a\right)$ ) except perhaps for a sequence $x_{n} \rightarrow x_{0}$ where $V_{x}^{-}\left(x_{n}\right) \neq V_{x}^{+}\left(x_{n}\right)$. In this last case, however, we have

$$
\lim _{\substack{x \uparrow_{0} \\ x \in\left(x_{0}-a, x_{0}\right]}} p(x)=V_{x}^{-}\left(x_{0}\right) \quad\left(\text { respectively } \lim _{\substack{x \downarrow x_{0} \\ x \in\left[x_{0}, x_{0}+a\right)}} p(x)=V_{x}^{+}\left(x_{0}\right)\right),
$$


where $p(x) \in\left\{V_{x}^{-}(x), V_{x}^{+}(x)\right\}$.

Proof. 1. The value function $V$ is the viscosity solution of the (OHJB) equation. If we write this equation in the form of (3.28) with $H$ given in (3.29), then, using that $\frac{x^{2}}{x^{2}+1}-0.6 x<0$ for $x \in(0,2)$, we have that

$$
H(x, \lambda) \rightarrow-\infty \quad \text { as } \quad \lambda \rightarrow-\infty .
$$

Now, for fixed $x \in(0,2)$ consider the function

$$
\phi(y)=V(y)+C|y-x|,
$$

where $C>0$ is a constant to be chosen later. The fact that $W$ is bounded and continuous in $(0,2)$ implies the existence of $\bar{y} \in(0,2)$ such that

$$
\phi(\bar{y})=\min _{y \in(0,2)} \phi(y) .
$$

Let $y_{1} \in(0,2), y_{1} \leq x$. Choosing $y_{2} \in(0,2), y_{2} \geq x$ with $\left|y_{2}-x\right| \leq\left|y_{1}-x\right|$ we have that

$$
\begin{aligned}
\phi\left(y_{2}\right) & =V\left(y_{2}\right)+C\left|y_{2}-x\right| \leq V\left(y_{2}\right)+C\left|y_{1}-x\right| \\
\leq V\left(y_{1}\right)+C\left|y_{1}-x\right| & =\phi\left(y_{1}\right),
\end{aligned}
$$

since $W$ is strictly decreasing in $(0,2)$. We deduce that, for every $y_{1} \in(0, x]$ there is $y_{2} \in[x, 2)$ such that $\phi\left(y_{2}\right) \leq \phi\left(y_{1}\right)$, so we have that $\bar{y} \geq x$.

The claim is that $\bar{y}=x$ for $C$ large. If not, then $\bar{y}>x$ and we would have

$$
\rho W(\bar{y})+H(\bar{y},-C) \geq 0
$$

since $W$ is a viscosity supersolution of (OHJB) and $y \rightarrow-C|y-x|$ is differentiable at $y=\bar{y}>x$. We have that $-C \rightarrow-\infty$ as $C \rightarrow+\infty$, so for sufficiently large $C$, independent of $x$, due to (3.32) we will have that

$$
\rho W(\bar{y})+H(\bar{y},-C)<0,
$$

which contradicts (3.33). Therefore, for such $C, x$ is the point where $\phi$ has its minimum, so

$$
\begin{aligned}
& \phi(x) \leq \phi(y) \quad \forall y \in(0,2) \\
\Rightarrow & W(x) \leq W(y)+C|y-x| \quad \forall y \in(0,2) \\
\Rightarrow & W(y)-W(x) \geq-C|y-x| \quad \forall y \in(0,2) .
\end{aligned}
$$

By interchanging the roles of $x$ and $y$ the proof is completed.

2. We can show, using simple calculus, that:

(i) For every $c, M \in \mathbb{R}$ the set

$$
H^{-1}(\{c\}) \cap([-M, M] \times[-M, 0))
$$

is either empty or finite.

(ii) The local extremum values (i.e. the local minimum and maximum values) of $H$ are isolated. 
Thus, we can prove the claim of the proposition following the proof of Theorem 1 of [13, with minor changes due to the fact that here $H$ is a function of two variables. So, we apply a similar "blow-up" argument to the equation

$$
\rho W(x)+H(x, D W)=0, \quad x \in(0,2) .
$$

More precisely, for $x_{0} \in(0,2)$ and $\delta>0$ sufficiently small, let $V^{\delta}, x^{\delta}:[-1,1] \rightarrow \mathbb{R}$ be defined by

$$
\begin{gathered}
V^{\delta}(x)=\frac{V\left(x_{0}+\delta x\right)-V\left(x_{0}\right)}{\delta} \text { and } \\
x^{\delta}(x)=x_{0}+\delta x .
\end{gathered}
$$

It turns out that $W^{\delta}$ is a viscosity solution of

$$
\delta \rho V^{\delta}+H\left(x^{\delta}, V_{x}^{\delta}\right)=-\rho V\left(x_{0}\right) \quad \text { in } \quad(-1,1) .
$$

So, focusing on a neighborhood of $x_{0}$, we pass from equation (3.34) to equation (3.35) building $V^{\delta}$, which possesses all the nice properties of $V$. Then we show that the family $\left\{V^{\delta}: \delta>0\right\}$ converges uniformly, along subsequences, to $V^{\infty}:[-1,1] \rightarrow \mathbb{R}$ and that $W^{\infty}$ inherits all the nice properties of $V^{\delta}$. From (3.35) we deduce that $W^{\infty}$ satisfies the equation

$$
H\left(x_{0}, V_{x}^{\infty}\right)=-\rho V\left(x_{0}\right) \text { in }(-1,1),
$$

which is the analogue of relation (0.5) of [13. Finally we use lemmas 1.2 and 1.3 of [13] (the fact that here $H$ is a two-variable function does not change their proofs significantly) to obtain that $V^{\infty}$ is unique and either linear or piecewise linear with only one corner at $x=0$, hence the existence of $V_{x}^{ \pm}\left(x_{0}\right)$. The first part of $(\mathrm{A})$ is immediate from the properties of $V^{\infty}$, and the second part is immediate from the definition of the viscosity solution.

3. The proof of this claim is similar to the proof of Theorem 2 of [13].

In the case of a Skiba point the welfare function constructed in Section 2 is the viscosity solution of the equation (OHJB). Following the notation of Section 2 we have the next theorem:

Theorem 3.9. If the system (2.7) has three steady-states with a Skiba point at $x=x_{s}$, we can construct the value function $V$ by using (2.9) with $V=W_{r}$ for $x \leq x_{s}$ and $V=W_{r}$ for $x \geq x_{s}$. The so-constructed $V$ is a viscosity solution of (OHJB) in $(0,2)$, with the proper boundary conditions. That is, $V=W$, where $W$ is the classically constructed in Section 2 welfare function.

Proof. Let us recall from Section 2 that the welfare function provided by relation (2.9) is multi-valued in $\left[x_{1}, x_{2}\right]$ (with $W_{l}$ and $W_{r}$ the two branches) and the Skiba point $x_{s}$ corresponds to the intersection of the two graphs $W_{l}$ and $W_{r}$ in this domain (see Fig. 5). We construct $V$ so that it is continuous and equals $W_{l}$ on the left of $x_{s}$ and $W_{r}$ on the right of $x_{s}$, that is,

$$
V= \begin{cases}W_{l}, & x \leq x_{s} \\ W_{r}, & x \geq x_{s}\end{cases}
$$

(see Fig. 17). Given the fact that the optimal trajectories of (2.7) cannot cross the positive 


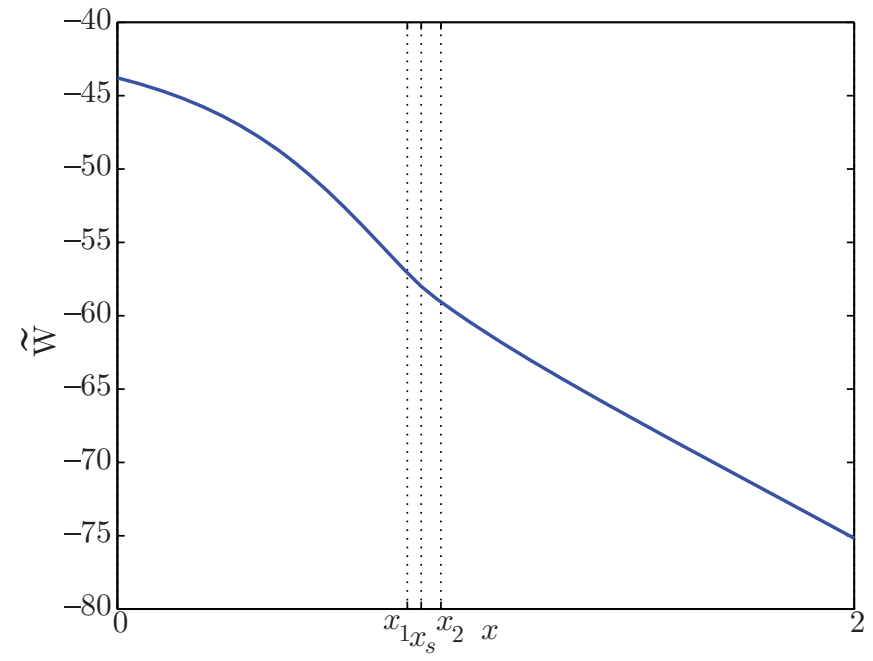

FIG. 7. The value function is chosen equal to $W_{l}$ on the left side of $x_{s}$ and to $W_{r}$ on the right side of $x_{s}\left(W_{l}\right.$ and $W_{r}$ are calculated by (2.9); see Fig. 5).

$x$-axis and do not approach $(0,0)$ (see [21) we have that $\alpha>0$. Hence $\lambda(t)<0$, and (2.4) and the first equation of (2.7) form the system of the characteristic equations for (OHJB). Therefore, $W_{l}$ and $W_{r}$, obtained by (2.9), are classical solutions of the (OHJB) equation for $x<x_{s}$ and $x>x_{r}$ respectively, that is,

$$
\left\{\begin{array}{l}
\rho W_{l}(x)+H\left(x, W_{l x}\right)=0 \\
\rho W_{r}(x)+H\left(x, W_{r_{x}}\right)=0,
\end{array}\right.
$$

where $H(x, \mu)$ is given by (3.29). What remains to be shown is that $V$ satisfies the viscosity criterion at the Skiba point $x_{s}$ (where there is a corner due to the jump of the derivative of $V$ at this point); see Theorem 1.3 of [9. From (2.10) we have $D^{+} V\left(x_{s}\right)=\emptyset$ and $D^{-} V\left(x_{s}\right)=\left[W_{l x}^{-}\left(x_{s}\right), W_{r x}^{+}\left(x_{s}\right)\right]$; see Fig. 7 So, according to condition (jj), we have to show that $\rho V\left(x_{s}\right)+H\left(x_{s}, \mu\right) \geq 0, \forall \mu \in\left[W_{l x}\left(x_{s}\right), W_{r x}\left(x_{s}\right)\right]$. But this is a direct consequence of the concavity of $H\left(x_{s}, \mu\right)$ with respect to $\mu$ and (3.37).

REMARK 3.10. In the case of one saddle point, the value function obtained by integration (2.9) is the classical solution obtained by means of characteristics.

Based on the result of Theorem 3.9 we have that the welfare function (3.36) is locally semiconvex and strictly decreasing on $(0,2)$. Some additional properties are mentioned in the next proposition:

Proposition 3.11. The welfare function $W$ has the following properties:

(1) $W$ is concave at $\left[0, x_{b}\right]$, where $x_{b}$ (which depends on $b$ ) is characterized as follows: If $x^{\prime}(t)=\alpha(t)-k(x(t))$ is the dynamical system of the specific optimal control problem, then $\left(0, x_{b}\right)$ is the maximal interval for which we have $k^{\prime}(x) \geq 0$ and $k^{\prime \prime}(x)<0$.

(2) $W$ is $C^{1}\left[0, x_{b}\right]$ (it is smooth in the region where it is concave). 
REMARK 3.12. From the properties of $x_{b}$ it is clear that this is the flip point of the lake, that is, the point at which the lake flips from an oligotrophic state to a eutrophic state, with a high equilibrium level of phosphorus and with low levels of ecological services.

REMARK 3.13. In general, if the welfare function is

$$
W\left(x_{0}\right)=\sup _{\alpha \in A} \int_{0}^{\infty} e^{-\rho t}[U(\alpha(t))-D(y(t))] d t
$$

and the dynamical system is in the form of Proposition 3.11, then assuming that

- $k$ is nondecreasing and concave at $\left(0, y_{0}\right)$,

- $U$ is strictly concave at $B$,

- $D$ is convex and nondecreasing at $\left(0, y_{0}\right)$, we have that $W$ is concave and $C^{1}$ at $\left[0, y_{0}\right]$ (see Proposition 1 and Theorem 1 of [14]).

Proof. We have that $k(x)=b x-\frac{x^{2}}{x^{2}+1}$ and, using calculus, we deduce that, for every $b \geq 0$ there is a point $x_{b}$ for which we have $k^{\prime}(x) \geq 0$ and $k^{\prime \prime}(x)<0$ for $x \in\left(0, x_{b}\right)$. The welfare function is

$$
W\left(x_{0}\right)=\max _{\left(\alpha_{1}, \alpha_{2}\right) \in A} \int_{0}^{\infty} e^{-\rho t}\left[\ln \left(\alpha_{1}(t)\right)+\ln \left(\alpha_{2}(t)\right)-2 y^{2}(t)\right] d t .
$$

If $U\left(a_{1}, a_{2}\right)=\ln \left(a_{1}\right)+\ln \left(a_{2}\right)$ and $D(x)=2 x^{2}$, then $U$ is strictly concave for every $\left(a_{1}, a_{2}\right) \in \mathbb{R}^{+} \times \mathbb{R}^{+}$(proved using the definition of concavity) and $D$ is convex and nondecreasing at $(0,+\infty)$. Using Proposition 1 and Theorem 1 of [14] (see Remark 3.13), we deduce claims 1 and 2 .

\section{Numerical investigation of the control problem by discretizing the vis- cosity solution.}

4.1. Numerical schemes. As we have explained in Section 2, the classical approach to obtaining the welfare function (1.3) is by first constructing the stable manifolds of the optimal dynamical system (2.7) and then by integrating the function $-\frac{2}{\alpha(x)}$; see (2.9). The scope of this section is to present an alternative, more robust, method of approximating numerically the welfare function through convergent monotone schemes by approximating the viscosity solution of (OHJB). This is justified by the result of Theorem 3.9. Given that the only boundary conditions available are the welfare values at the saddle points given by (2.8), we construct three different finite difference schemes. The "forward" scheme is given by

$$
\left\{\begin{array}{l}
W_{i+1}=\Delta x \cdot \frac{\rho W_{i+1}+2\left(\ln \left(-\frac{W_{i+1}-W_{i}}{\Delta x}\right)+x_{i+1}{ }^{2}+1\right)}{\frac{x_{i+1}{ }^{2}}{x_{i+1}+1}-0.6 x_{i+1}}+W_{i}, \quad i=0,1, \ldots, N-1, \\
W_{0}=W\left(x_{s 0}\right),
\end{array}\right.
$$

where $\Delta x=\frac{2-x_{s 0}}{N}$ and $x_{i+1}=x_{i}+\Delta x, i=0, \ldots, N-1 \quad\left(x_{0}=x_{s 0}\right)$. The "backward" scheme is given by

$$
\left\{\begin{array}{l}
W_{i}=\Delta y \cdot e^{\frac{\left(\frac{x_{i}{ }^{2}}{x_{i}^{2}+1}-0.6 x_{i}\right)\left(W_{i+1}-W_{i}\right)-\rho \Delta y \cdot W_{i}-2\left(x_{i}{ }^{2}+1\right) \Delta y}{2 \Delta y}}+W_{i+1}, \quad i=-1,-2, \ldots,-M, \\
W_{0}=W\left(x_{s 0}\right)
\end{array}\right.
$$


where $\Delta y=\frac{x_{s 0}-0}{M}$ and $x_{i-1}=x_{i}-\Delta y, i=0,-1, \ldots,-M+1 \quad\left(x_{0}=x_{s 0}\right)$. In these two schemes, we approximate $W\left(x_{i}\right)$ with $W_{i}, i=-M, \ldots, 0,1, \ldots, N$. The "upwind" scheme is given by

$$
\left\{\begin{array}{l}
W_{i}^{(l+1)}=\frac{a_{i} \frac{W_{i}^{(l+1)}-W_{i-1}^{(l)}}{\Delta z}-2\left(\ln \left(-\frac{W_{i+1}^{(l)}-W_{i}^{(l+1)}}{\Delta z}\right)+\beta_{i}\right)}{\rho}, \quad i=1,2, \ldots, L-1, \quad l=0,1,2, \ldots, \\
W_{0}^{(r)}=W\left(x_{s 1}\right), \quad W_{L}^{(r)}=W\left(x_{s 2}\right), \quad r=0,1,2, \ldots, \\
W_{i}^{(0)}, i=1,2, \ldots, L-1 \quad \text { is linear interpolation of } W_{0}^{(0)}, W_{L}^{(0)},
\end{array}\right.
$$

where $\Delta z=\frac{x_{s 2}-x_{s 1}}{L}$.

In the case of one saddle point $x_{s 0} \in(0,2)$, we use the backward scheme in $\left(0, x_{s 0}\right]$ and the forward scheme in $\left[x_{s 0}, 2\right]$. In the case of two saddle points $x_{s 1}, x_{s 2} \in(0,2)$, we use the upwind scheme in $\left[x_{s 1}, x_{s 2}\right]$ and the forward and backward schemes in the intervals $\left[x_{s 2}, 2\right)$ and $\left(0, x_{s 1}\right]$, respectively.

The above three implicit schemes can be iteratively solved, and their convergence is guaranteed by fixed-point arguments which are presented in Section4.2. We next explain the way the above numerical schemes are derived.

For the forward scheme, the derivative $D W\left(x_{i+1}\right)$ in (OHJB) is replaced by the backward finite-difference approximation $D W\left(x_{i+1}\right) \simeq \frac{W\left(x_{i+1}\right)-W\left(x_{i}\right)}{\Delta x}$, so (OHJB) at $x_{i+1}$ is written in the discrete form,

$$
\rho W_{i+1}=\left(\frac{x_{i+1}^{2}}{x_{i+1}{ }^{2}+1}-0.6 x_{i+1}\right) \frac{W_{i+1}-W_{i}}{\Delta x}-2\left(\ln \left(-\frac{W_{i+1}-W_{i}}{\Delta x}\right)+x_{i+1}^{2}+1\right) .
$$

By solving with respect to $W_{i+1}$, in the first term of the right-hand side in the above equation, we obtain (4.1). For the backward scheme, the derivative $D W\left(x_{i}\right)$ in (OHJB) is replaced by the forward finite-difference approximation $D W\left(x_{i}\right) \simeq \frac{W\left(x_{i+1}\right)-W\left(x_{i}\right)}{\Delta y}$, so (OHJB) at $x_{i}$ is written in the discrete form

$$
\rho W_{i}=\left(\frac{x_{i}{ }^{2}}{x_{i}{ }^{2}+1}-0.6 x_{i}\right) \frac{W_{i+1}-W_{i}}{\Delta y}-2\left(\ln \left(-\frac{W_{i+1}-W_{i}}{\Delta y}\right)+x_{i}{ }^{2}+1\right) .
$$

By solving with respect to $W_{i}$, in the second term of the right-hand side of the above equation, we obtain (4.2).

For the upwind scheme, the derivative $D W\left(x_{i}\right)$ in the first term of the right-hand side of (OHJB) is replaced by the backward finite-difference approximation $D W\left(x_{i}\right) \simeq$ $\frac{W\left(x_{i}\right)-W\left(x_{i-1}\right)}{\Delta z}$, while the derivative in the second term is replaced by the forward finitedifference approximation $D W\left(x_{i}\right) \simeq \frac{W\left(x_{i+1}\right)-W\left(x_{i}\right)}{\Delta z}$. So, (OHJB) at $x_{i}$ is written in the discrete form

$$
\rho W_{i}=\left(\frac{x_{i}^{2}}{x_{i}{ }^{2}+1}-0.6 x_{i}\right) \frac{W_{i}-W_{i-1}}{\Delta z}-2\left(\ln \left(-\frac{W_{i+1}-W_{i}}{\Delta z}\right)+x_{i}{ }^{2}+1\right) .
$$

By solving with respect to $W_{i}$ in the left-hand side of the above equation and setting $a_{i}=\frac{x_{i}{ }^{2}}{x_{i}{ }^{2}+1}-0.6 x_{i}$ and $\beta_{i}=x_{i}{ }^{2}+1$, we obtain (4.3).

We now have the following convergence theorem:

ThEOREM 4.1. The numerical schemes derived above are consistent and monotone. In addition, they are stable and converge to the correct viscosity solution limit. 
Proof. The proof of this theorem consists of the following steps:

- First, we show that the numerical schemes (4.1), (4.2) and (4.3) are consistent. This is obtained by the standard method for finite differences. We write scheme (4.1) with $W\left(x_{i+1}\right)$ in place of $W_{i+1}$, so we have an error $r_{i+1}$ added at the end of this equation. Subtracting this from equation (OHJB) and using the Taylor expansion and inequality $\ln (x) \leq x-1, x \in \mathbb{R}$, we deduce that

$$
\max _{0 \leq i \leq N-1}\left|r_{i+1}\right| \leq C h^{2},
$$

where $C$ is a constant. The same approach is followed for schemes (4.2), (4.3), as well. This relation shows the consistency of the schemes.

- The numerical scheme (4.1) is an approximation scheme of the form

$$
S\left(r, x, u^{r}(x), u^{r}\right)=0 \quad \text { in } \bar{\Omega},
$$

where $S: \mathbb{R}^{+} \times \bar{\Omega} \times \mathbb{R} \times B(\bar{\Omega}) \rightarrow \mathbb{R}$ is locally bounded $\left(\mathbb{R}^{+} \equiv[0, \infty), \Omega \subseteq \mathbb{R}^{n}\right.$ is open and bounded and $B(\bar{\Omega})$ is the space of bounded functions defined in $\bar{\Omega}$; see [4]). Here, $r=\Delta x, x=x_{i+1}$ and $u^{r}$ is a function defined on $J^{\Delta x}=$ $\left\{x_{i}: i=0, \ldots, N\right\}$ by $u_{i+1}^{r}=W_{i+1}$. Then, numerical scheme (4.1) is written as

$$
S\left(\Delta x, x_{i+1}, W_{i+1}, u^{r}\right)=0,
$$

where

$$
S(k, y, t, u)=t-u_{i}-k \frac{\rho t+2\left[\ln \left(-\frac{t-u_{i}}{k}\right)+y^{2}+1\right]}{\frac{y^{2}}{y^{2}+1}-0.6 y},
$$

with $k \geq 0, y \in J^{\Delta x}, t \in \mathbb{R}$ and $u \in B\left(J^{\Delta x}\right)$. The numerical schemes (4.2), (4.3) are treated in an analogous way.

- The numerical scheme (4.1) is monotone. For this we must show that

$$
S(k, y, t, u) \leq S(k, y, t, w) \quad \text { if } u \geq w,
$$

for all $k \geq 0, y \in J^{\Delta x}, t \in \mathbb{R}$ and $u, w \in B\left(J^{\Delta x}\right)$. We have

$$
\frac{d S}{d u_{i}}=-1-\frac{2 k}{\frac{y^{2}}{y^{2}+1}-0.6 y}\left(-\frac{k}{t-u_{i}}\right) \frac{1}{k}=-1+\frac{2 k}{\left(\frac{y^{2}}{y^{2}+1}-0.6 y\right)\left(t-u_{i}\right)} \text {. }
$$

We will impose the following a priori restriction for $W_{i}, i=0, \ldots, N$, which we will also use in the sequel,

$$
W_{i+1}-W_{i}<\frac{(2+\varepsilon) \Delta x}{\frac{x_{i+1^{2}}}{x_{i+1}{ }^{2}+1}-0.6 x_{i+1}},
$$

$\varepsilon>0$ small. Therefore,

$$
\begin{aligned}
& t-u_{i}<\frac{(2+\varepsilon) k}{\frac{y^{2}}{y^{2}+1}-0.6 y} \Rightarrow \frac{1}{t-u_{i}}>\frac{\frac{y^{2}}{y^{2}+1}-0.6 y}{(2+\varepsilon) k} \\
\Rightarrow \quad & \frac{2 k}{\left(\frac{y^{2}}{y^{2}+1}-0.6 y\right)\left(t-u_{i}\right)}<\frac{2}{2+\varepsilon}<1 \Rightarrow \frac{2 k}{\left(\frac{y^{2}}{y^{2}+1}-0.6 y\right)\left(t-u_{i}\right)}-1<0 .
\end{aligned}
$$


From (4.4) we deduce that scheme (4.1) is monotone. The numerical schemes (4.2), (4.3) are studied in an analogous way.

- Consistent and monotone schemes for the HJ equations are stable and converge to the correct viscosity solution (see 4]; 17, page 104).

The theorem is proved.

4.2. Iterative method. The numerical schemes introduced in the previous section are implicit. An iterative method is needed to compute $W_{i}$. Such methods are presented in this section. We first consider the cases of the numerical schemes (4.1), (4.2). We have the following theorem:

Proposition 4.2. The functions $g_{1}:\left[W_{l b}, W_{i}+\frac{(2+\varepsilon) \Delta x}{a_{i+1}}\right] \rightarrow\left[W_{l b}, W_{i}+\frac{(2+\varepsilon) \Delta x}{a_{i+1}}\right]$, where

$$
g_{1}(x)=\frac{\Delta x}{a_{i+1}}\left(\rho x+2\left[\ln \left(-\frac{x-W_{i}}{\Delta x}\right)+\beta_{i+1}\right]\right)+W_{i}
$$

for $i=0,1, \ldots, N-1$ and $g_{2}:\left[W_{i+1}, W_{i+1}-\frac{2 \Delta y}{a_{i}}\right] \rightarrow\left[W_{i+1}, W_{i+1}-\frac{2 \Delta y}{a_{i}}\right]$, where

$$
g_{2}(x)=\Delta y \cdot e^{\frac{a_{i}\left(W_{i+1}-x\right)-\rho \Delta y \cdot x-2 \beta_{i} \Delta y}{2 \Delta y}}+W_{i+1}
$$

for $i=-1,-2, \ldots,-M$, where $a_{i+1}=\frac{x_{i+1}{ }^{2}}{x_{i+1}{ }^{2}+1}-0.6 x_{i+1}, \quad \beta_{i+1}=x_{i+1}{ }^{2}+1, i=$ $-M-1, \ldots, 0,1, \ldots, N-1$, are contraction mappings for specific $\Delta x, \Delta y, \varepsilon>0$ small enough, with $W_{l b}$ the lower bound of $W_{i}, i=-M, \ldots, 0,1, \ldots, N$, if the following conditions are satisfied:

- $\rho \leq \frac{k+(2+\varepsilon)-2 \ln \left(-\frac{2+\varepsilon}{a_{i+1}}\right)-2 \beta_{i+1}}{W_{i}} \quad(\varepsilon, k>0$ arbitrary small $)$, $i=0, \ldots, N-1$,

- $\rho<\min \left\{\frac{(-2)\left[\ln \left(-\frac{2}{a_{i}}\right)+1-\beta_{i}\right]}{W_{i+1}},-\frac{a_{i}}{\Delta y}\right\}, \quad i=-M, \ldots,-1$,

- $\Delta x \leq-\frac{a_{i+1}}{\rho} \cdot \frac{2 \ln \left(-\frac{2+\varepsilon}{a_{i+1}}\right)+2 \beta_{i+1}-(2+\varepsilon)+\rho W_{i}}{2+\varepsilon}, \quad i=0, \ldots, N-1$,

- $W_{l b}=\frac{\rho W_{0}-2}{\rho}$.

Then, $W_{i+1}{ }^{(k+1)}=g_{1}\left(W_{i+1}{ }^{(k)}\right) \in\left[W_{l b}, W_{i}+\frac{(2+\varepsilon) \Delta x}{a_{i+1}}\right], \forall k \in \mathbb{N}, i=0,1, \ldots, N-1$, with $W_{i+1}{ }^{(0)}=W_{i}+\frac{(2+\varepsilon) \Delta x}{a_{i+1}}$ and the sequence $W_{i+1}{ }^{(k+1)}$ converges to a unique $W_{i+1}$ and $W_{i}{ }^{(k+1)}=g_{2}\left(W_{i}^{(k)}\right) \in\left[W_{i+1}, W_{i+1}-\frac{2 \Delta y}{a_{i}}\right], \forall k \in \mathbb{N}, i=-1,-2, \ldots,-M$, with $W_{i}{ }^{(0)}=W_{i+1}$ and the sequence $W_{i}{ }^{(k+1)}$ converges to a unique $W_{i}$, as long as the following inequalities hold:

- $\left|W_{i+1}-W_{i}\right|>-\frac{(2+\varepsilon) \Delta x}{a_{i+1}}, i=0,1, \ldots, N-1$, and

$\left|W_{i+1}-W_{i}\right| \leq-\frac{2 \Delta y}{a_{i}}, i=-1,-2, \ldots,-M$,

- $W_{N}-W_{l b} \geq-\frac{25 \Delta x}{\max \left\{a_{i+1}\right\}}>0$.

Proof. We will prove the result for the forward scheme, i.e. for function $g_{1}$. We take $x, y \in\left[W_{l b}, W_{i}+\frac{(2+\varepsilon) \Delta x}{a_{i+1}}\right]$ with $x<y$. Then

$$
g_{1}(x)-g_{1}(y)=\frac{\Delta x}{a_{i+1}}\left(\rho(x-y)+2 \ln \left(\frac{-x+W_{i}}{-y+W_{i}}\right)\right) .
$$


If $f(x)=\frac{x^{2}}{x^{2}+1}-0.6 x$, then, using calculus, we deduce that $f(x)<0, \forall x>0$. So,

$$
a_{i}<0, \quad i=0, \ldots, N .
$$

We will next prove that $g_{1}(x)$ is increasing in $\left[W_{l b}, W_{i}+\frac{(2+\varepsilon) \Delta x}{a_{i+1}}\right]$. We have $g_{1}{ }^{\prime}(x)=$ $\frac{\rho\left(x-W_{i}\right) \Delta x+2 \Delta x}{a_{i+1}\left(x-W_{i}\right)}$ and

$$
\begin{aligned}
\rho\left(x-W_{i}\right) \Delta x+2 \Delta x & =\Delta x\left(\rho\left(x-W_{i}\right)+2\right) \geq \Delta x\left(\rho\left(W_{l b}-W_{0}\right)+2\right) \\
& =\Delta x\left(\rho\left(\frac{\rho W_{0}-2}{\rho}-W_{0}\right)+2\right) \text { by }(\mathrm{D}) \\
& =\Delta x\left(\rho W_{0}-2-W_{0} \rho+2\right)=0 .
\end{aligned}
$$

Hence,

$$
\rho\left(x-W_{i}\right) \Delta x+2 \Delta x \geq 0 .
$$

In addition, due to (4.6),$a_{i+1}\left(x-W_{i}\right)>0$, so $g_{1}{ }^{\prime}(x) \geq 0$ in $\left[W_{l b}, W_{i}+\frac{(2+\varepsilon) \Delta x}{a_{i+1}}\right]$. Using inequality $\ln (x) \leq x-1, x \in \mathbb{R}^{+}$, we have from (4.5), (4.6) that

$$
\begin{aligned}
g_{1}(x)-g_{1}(y) & \geq \frac{2 \Delta x}{a_{i+1}} \ln \left(\frac{-x+W_{i}}{-y+W_{i}}\right) \geq \frac{2 \Delta x}{a_{i+1}}\left(\frac{-x+W_{i}}{-y+W_{i}}-1\right) \\
& =\frac{2 \Delta x}{a_{i+1}}\left(\frac{y-x}{-y+W_{i}}\right) .
\end{aligned}
$$

Since the function $g_{1}$ is increasing, we have

$$
\left|g_{1}(x)-g_{1}(y)\right| \leq-\frac{2 \Delta x}{a_{i+1}\left(-y+W_{i}\right)}|y-x| .
$$

If $m(x)=-\frac{2 \Delta x}{a_{i+1}\left(-x+W_{i}\right)}$, then $m^{\prime}(x)=-\frac{2 \Delta x}{a_{i+1}}\left(\frac{1}{\left(-x+W_{i}\right)^{2}}\right)>0$ for $x \in\left[W_{l b}, W_{i}+\frac{(2+\varepsilon) \Delta x}{a_{i+1}}\right]$, due to (4.6). So, $y \leq W_{i}+\frac{(2+\varepsilon) \Delta x}{a_{i+1}}$ and

$$
m(y) \leq m\left(W_{i}+\frac{(2+\varepsilon) \Delta x}{a_{i+1}}\right)=-\frac{2 \Delta x}{a_{i+1}\left(-W_{i}-\frac{(2+\varepsilon) \Delta x}{a_{i+1}}+W_{i}\right)}=\frac{2}{2+\varepsilon} .
$$

Therefore, from (4.7) using (4.8) we have

$$
\left|g_{1}(x)-g_{1}(y)\right| \leq \frac{2}{2+\varepsilon}|x-y| .
$$

If $x>y$ following the previous techniques, we end up with the same inequality. So we have that

$$
\left|g_{1}(x)-g_{1}(y)\right| \leq \frac{2}{2+\varepsilon}|x-y| \quad \forall x, y \in\left[W_{l b}, W_{i}+\frac{(2+\varepsilon) \Delta x}{a_{i+1}}\right] .
$$

We conclude that $g_{1}$ is a contraction in $\left[W_{l b}, W_{i}+\frac{(2+\varepsilon) \Delta x}{a_{i+1}}\right]$. We next show that

$$
g_{1}\left(\left[W_{l b}, W_{i}+\frac{(2+\varepsilon) \Delta x}{a_{i+1}}\right]\right) \subseteq\left[W_{l b}, W_{i}+\frac{(2+\varepsilon) \Delta x}{a_{i+1}}\right] .
$$

Since $g_{1}$ is increasing and continuous, it is sufficient to show that

$$
\left[g_{1}\left(W_{l b}\right), g_{1}\left(W_{i}+\frac{(2+\varepsilon) \Delta x}{a_{i+1}}\right)\right] \subseteq\left[W_{l b}, W_{i}+\frac{(2+\varepsilon) \Delta x}{a_{i+1}}\right] .
$$


For this, it is sufficient to show that

$$
g_{1}\left(W_{l b}\right) \geq W_{l b}
$$

and

$$
g_{1}\left(W_{i}+\frac{(2+\varepsilon) \Delta x}{a_{i+1}}\right) \leq W_{i}+\frac{(2+\varepsilon) \Delta x}{a_{i+1}}
$$

(then, $g_{1}\left(W_{i}+\frac{(2+\varepsilon) \Delta x}{a_{i+1}}\right) \geq g_{1}\left(W_{l b}\right) \geq W_{l b}$ and $g_{1}\left(W_{l b}\right) \leq g_{1}\left(W_{i}+\frac{(2+\varepsilon) \Delta x}{a_{i+1}}\right) \leq W_{i}+$ $\left.\frac{(2+\varepsilon) \Delta x}{a_{i+1}}\right)$. Relation (4.10) is written as

$$
\frac{\Delta x}{a_{i+1}}\left(\rho W_{l b}+2\left[\ln \left(-\frac{W_{l b}-W_{i}}{\Delta x}\right)+\beta_{i+1}\right]\right)+W_{i} \geq W_{l b} .
$$

Hence,

$$
2 \ln \left(\frac{W_{i}-W_{l b}}{\Delta x}\right)+a_{i+1} \frac{W_{i}-W_{l b}}{\Delta x}+\rho W_{l b}+2 \beta_{i+1} \leq 0 .
$$

If $h(x)=2 \ln (x)+a_{i+1} x+10, x>0$, then $h$ has a global maximum at $x_{0}=-\frac{2}{a_{i+1}}$ and is decreasing for $x \geq x_{0}$. In addition, $\lim _{x \rightarrow+\infty} h(x)=-\infty$. If $h\left(x_{0}\right) \leq 0$, then $h(x) \leq 0, \forall x>0$, and (4.12) holds (as the left part of it is smaller than $h(x)$ ). If $h\left(x_{0}\right)>0$, then we have: Assuming that $h\left(-\frac{25}{a_{i+1}}\right) \geq 0$, then

$$
\begin{aligned}
& 2 \ln \left(-\frac{25}{a_{i+1}}\right)+a_{i+1}\left(-\frac{25}{a_{i+1}}\right)+10 \geq 0 \Rightarrow \ln \left(-\frac{25}{a_{i+1}}\right) \geq 7.5 \\
\Rightarrow & -\frac{25}{a_{i+1}} \geq e^{7.5} \Rightarrow a_{i+1} \geq-\frac{25}{e^{7.5}} \approx-0.014,
\end{aligned}
$$

which is a contradiction, since we can easily see that $\max _{0 \leq i \leq N}\left\{a_{i}\right\} \approx-0.0897$. So, $h\left(-\frac{25}{a_{i+1}}\right)<0$ and $-\frac{25}{a_{i+1}}>x_{0}$; hence $h(x)<0, \forall x \geq-\frac{25}{a_{i+1}}$. That is, (4.12) holds if $\frac{W_{i}-W_{l b}}{\Delta x} \geq-\frac{25}{a_{i+1}}$. The last inequality holds, since

$$
\frac{W_{i}-W_{l b}}{\Delta x} \geq \frac{W_{N}-W_{l b}}{\Delta x} \stackrel{(E 2)}{\geq}-\frac{25}{\max \left\{a_{i+1}\right\}} \geq-\frac{25}{a_{i+1}} .
$$

So, (4.12) holds and so does (4.10). Relation (4.11) is written as

$$
\begin{aligned}
& \frac{\Delta x}{a_{i+1}}\left(\rho W_{i}+\frac{(2+\varepsilon) \rho \Delta x}{a_{i+1}}+2\left[\ln \left(-\frac{2+\varepsilon}{a_{i+1}}\right)+\beta_{i+1}\right]\right)+W_{i} \\
& \leq W_{i}+\frac{(2+\varepsilon) \Delta x}{a_{i+1}} .
\end{aligned}
$$

Hence,

$$
2 \ln \left(-\frac{2+\varepsilon}{a_{i+1}}\right)+\frac{(2+\varepsilon) \rho \Delta x}{a_{i+1}}+\rho W_{i}+2 \beta_{i+1}-(2+\varepsilon) \geq 0 .
$$

Using condition $(\mathrm{C})$, the left-hand side of the above inequality is greater than

$$
\begin{aligned}
& 2 \ln \left(-\frac{2+\varepsilon}{a_{i+1}}\right)+(2+\varepsilon)\left(\frac{-2 \ln \left(-\frac{2+\varepsilon}{a_{i+1}}\right)-2 \beta_{i+1}+(2+\varepsilon)-\rho W_{i}}{2+\varepsilon}\right) \\
& +\rho W_{i}+2 \beta_{i+1}-(2+\varepsilon),
\end{aligned}
$$


which is equal to 0 . We thus conclude that (4.11) holds. So, relation (4.9) holds and we have shown that $g_{1}:\left[W_{l b}, W_{i}+\frac{(2+\varepsilon) \Delta x}{a_{i+1}}\right] \rightarrow\left[W_{l b}, W_{i}+\frac{(2+\varepsilon) \Delta x}{a_{i+1}}\right]$ is a contraction mapping for $i=0,1, \ldots, N-1$. Therefore, $g_{1}$ has a unique fixed point, the $W_{i+1}$, in this domain. ( $W_{i+1}$ is a fixed point for $g_{1}$, due to (4.1) and $W_{i+1} \in\left[W_{l b}, W_{i}+\frac{(2+\varepsilon) \Delta x}{a_{i+1}}\right]$, due to (E1)). In addition, $W_{i+1}{ }^{(k+1)}=g_{1}\left(W_{i+1}{ }^{(k)}\right) \in\left[W_{l b}, W_{i}+\frac{(2+\varepsilon) \Delta x}{a_{i+1}}\right], \forall k \in \mathbb{N}, i=$ $0,1, \ldots, N-1$ with $W_{i+1}{ }^{(0)}=W_{i}+\frac{(2+\varepsilon) \Delta x}{a_{i+1}}$ and the iterative scheme converges to the unique fixed point.

In a very similar way and using condition (B) instead of (A) and (C), it can be proved that $g_{2}:\left[W_{i+1}, W_{i+1}-\frac{2 \Delta y}{a_{i}}\right] \rightarrow\left[W_{i+1}, W_{i+1}-\frac{2 \Delta y}{a_{i}}\right]$ is a contraction mapping for $i=$ $-1,-2, \ldots,-M$, and that, similarly, $W_{i}^{(k+1)}=g_{2}\left(W_{i}^{(k)}\right) \in\left[W_{i+1}, W_{i+1}-\frac{2 \Delta y}{a_{i}}\right], \forall k \in$ $\mathbb{N}, i=-1,-2, \ldots,-M$, with $W_{i}^{(0)}=W_{i+1}$ and the iterative scheme converges to the unique fixed point.

REMARK 4.3. Condition (A) shows that

$$
\rho W_{i}-(2+\varepsilon)+2 \ln \left(-\frac{2+\varepsilon}{a_{i+1}}\right)+2 \beta_{i+1} \geq k, \quad k>0 \text { small }
$$

so it ensures that the right part of $(\mathrm{C})$ is positive and we can select $\Delta x$ small enough so that (C) is satisfied. In addition, it is easily seen that, for $k, \varepsilon>0$ small enough, the right part of (A) is positive, so we can find $\rho>0$ with (A) satisfied.

So in the case of one saddle point, the iterative methods are summarized as follows:

$$
\left\{\begin{array}{l}
W_{i+1}{ }^{(k+1)}=\frac{\Delta x}{a_{i+1}}\left(\rho W_{i+1}{ }^{(k)}+2\left[\ln \left(-\frac{W_{i+1}{ }^{(k)}-W_{i}}{\Delta x}\right)+\beta_{i+1}\right]\right)+W_{i}, \\
W_{i+1}{ }^{(0)}=W_{i}+\frac{(2+\varepsilon) \Delta x}{a_{i+1}}, \quad i=0,1, \ldots, N-1
\end{array}\right.
$$

for the forward scheme and

$$
\left\{\begin{array}{l}
W_{i}^{(k+1)}=\Delta y \cdot e^{\frac{a_{i}\left(W_{i+1}-W_{i}^{(k)}\right)-\rho \Delta y \cdot W_{i}(k)-2 \beta_{i} \Delta y}{2 \Delta y}}+W_{i+1} \\
W_{i}{ }^{(0)}=W_{i+1}, \quad i=-1,-2, \ldots,-M
\end{array}\right.
$$

for the backward one. From the previous theorem we have that

$$
\lim _{k \rightarrow \infty} W_{i+1}{ }^{(k)}=W_{i+1}, \quad i=-M-1, \ldots, 0,1, \ldots, N-1 .
$$

For the case of two saddle points, we have the following theorem:

Proposition 4.4. The finite difference equation (4.3) has a unique solution with respect to $W_{i}^{(l+1)}$ if $\Delta z$ satisfies the following condition:

$$
\Delta z \leq \min _{i=1, \ldots, L-1}\left\{\left(W_{i-1}^{(l)}-W_{i+1}^{(l)}\right) e^{\frac{\rho \cdot W_{i-1}^{(l)}}{2}+\beta_{i}}\right\} \quad \text { for } \quad l=0,1,2, \ldots
$$

Proof. It is sufficient to show that the function

$$
g_{3}(x)=\frac{1}{\rho}\left[\frac{a_{i}}{\Delta z}\left(x-W_{i-1}\right)-2\left(\ln \left(-\frac{W_{i+1}-x}{\Delta z}\right)+\beta_{i}\right)\right],
$$


where $a_{i}=\frac{x_{i}{ }^{2}}{x_{i}{ }^{2}+1}-0.6 x_{i}$ and $\beta_{i}=x_{i}{ }^{2}+1$, has a unique fixed point in $\left(W_{i+1}, W_{i-1}\right)$. The function $g_{3}$ is strictly decreasing in this domain $\left(g_{3}{ }^{\prime}(x)=\frac{a_{i}\left(x-W_{i+1}\right)-2 \Delta z}{\rho \Delta z\left(x-W_{i+1}\right)}<0\right.$ in $\left(W_{i+1}, W_{i-1}\right)$, as $a_{i}<0$ and $W$ is strictly decreasing) and $\lim _{x \rightarrow W_{i+1}} g_{3}(x)=+\infty$. Therefore, it is sufficient to show that $g_{3}\left(W_{i-1}\right) \leq W_{i-1}$. From (4.15), the following inequality holds for all $i=1, \ldots, L-1$ :

$$
\Delta z \leq\left(W_{i-1}-W_{i+1}\right) e^{\frac{\rho \cdot W_{i-1}}{2}+\beta_{i}} .
$$

The above equation is written in the equivalent form,

$$
-\frac{2}{\rho}\left(\ln \left(\frac{W_{i-1}-W_{i+1}}{\Delta z}\right)+\beta_{i}\right) \leq W_{i-1} .
$$

Hence, $g_{3}\left(W_{i-1}\right) \leq W_{i-1}$ and $g_{3}$ has a unique fixed point in $\left(W_{i+1}, W_{i-1}\right)$.

The unique solution of this scheme can be calculated using the Bisection Method.

5. Comparison with the simple shooting method. The Simple Shooting Method (see [20]) has been applied to our problem for both cases described above, with $b=0.6$. The values $W_{1}$ that this method produces were compared to the values $W_{2}$ produced by (4.1), (4.2) and (4.3). If we denote by $x_{i}, i=1, \ldots, N$, the points of discretization in the comparison domain, then, the Absolute Error (AE) and the Relative Error (RE) at $x_{i}$ are defined as follows:

$$
A E=\left|W_{1}\left(x_{i}\right)-W_{2}\left(x_{i}\right)\right| \quad \text { and } \quad R E=\frac{A E}{\left|W_{1}\left(x_{i}\right)\right|}, \quad i=1, \ldots, N .
$$

For $\rho=0.03$ we have one steady-state, saddle-stable, which is $(0.3534,0.1010)$. The initial condition for $W$ is $W(0.3534)=-207.369$. For $\Delta x=\Delta y=0.03$, the mean and the max $\mathrm{RE}$ between the two methods are $0.06 \%$ and $0.15 \%$, respectively (see Table 1 and Fig. (8).

TABLE 1. Results of the comparison between the Simple Shooting Method and the corresponding, depending on the case, numerical scheme. Mean and maximum values over the discretization points for the Absolute Error (AE) and for the Relative Error (RE) are provided.

\begin{tabular}{cccc}
\hline \hline No. of steady-states & Step & Error & {$[$ mean,max $]$} \\
\hline one & $\Delta x=\Delta y=0.03$ & $\mathrm{AE}$ & {$[0.150,0.332]$} \\
one & $\Delta x=\Delta y=0.03$ & $\mathrm{RE}(\%)$ & {$[0.06,0.15]$} \\
three & $\Delta z=0.01$ & $\mathrm{AE}$ & {$[0.081,0.199]$} \\
three & $\Delta z=0.01$ & $\mathrm{RE}(\%)$ & {$[0.15,0.34]$} \\
three & $\Delta z=0.001$ & $\mathrm{AE}$ & {$[0.011,0.069]$} \\
three & $\Delta z=0.001$ & $\mathrm{RE}(\%)$ & {$[0.02,0.12]$} \\
\hline \hline
\end{tabular}

For $\rho=0.13$ we have three steady-states (two saddle points and a vortex). The two saddle points are $(0.4783,0.1008)$ and $(1.2465,0.1395)$ and the vortex is $(0.8366,0.0902)$. The two boundary conditions are $W(0.4783)=-49.4852$ and $W(1.2465)=-64.8708$. The stopping criterion we used when applying the algorithm was $\left\|W-W^{\prime}\right\|_{\infty}<5 \cdot 10^{-4}$, where $W^{\prime}$ is the result 100 iterations after the result $W$. For $\Delta z=0.01$ the algorithm 


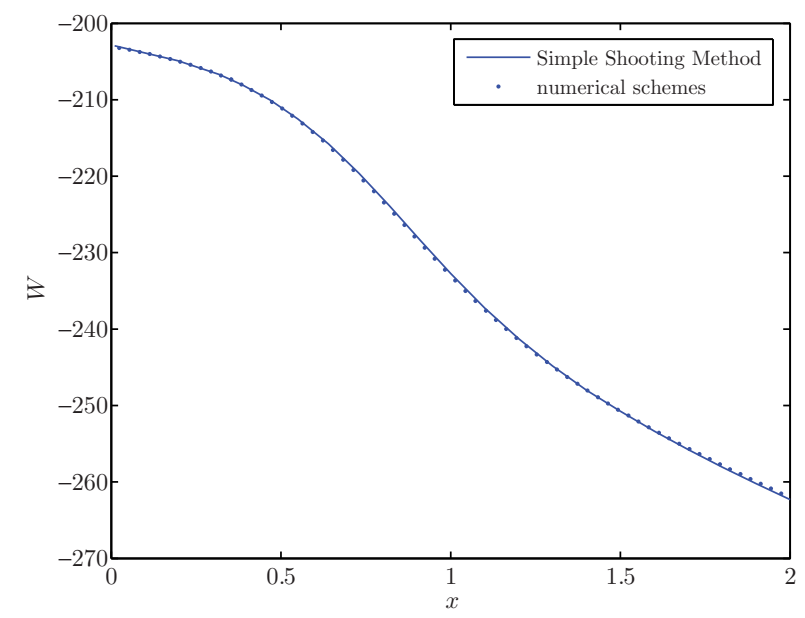

FiG. 8. Comparison between the Simple Shooting Method and the result of the numerical schemes in the one steady-state case $(b=$ $0.6, \rho=0.03, \Delta x=\Delta y=0.03)$.

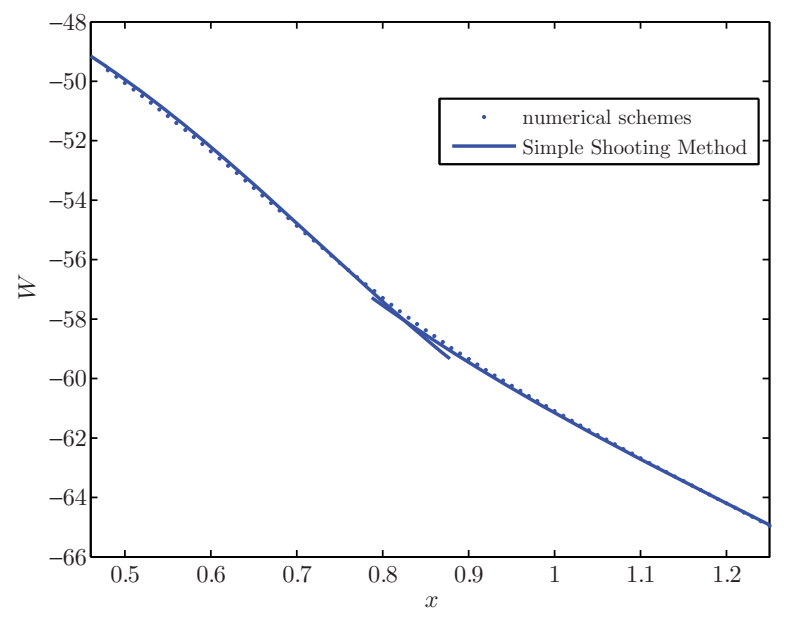

FIG. 9. Comparison between the Simple Shooting Method and the result of the numerical schemes in the three steady-states case $(b=$ $0.6, \rho=0.13, \Delta z=0.01)$.

stopped after 1000 iterations. The mean and the max RE between the two methods for this $\Delta z$ are $0.15 \%$ and $0.34 \%$, respectively (see Table 1 and Fig. 9 for this comparison). For $\Delta z=0.001,7100$ iterations are required and the results are much better: The above two errors are $0.02 \%$ and $0.12 \%$, respectively. 


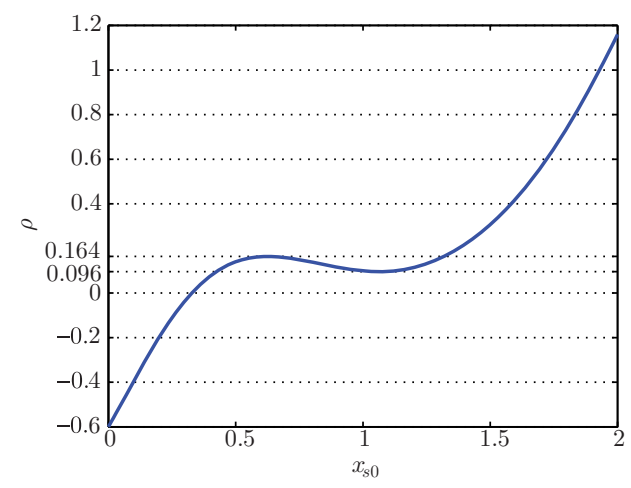

(a)

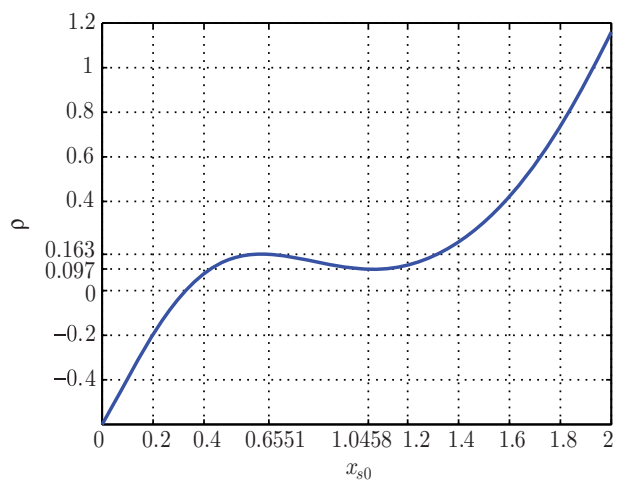

(b)

FIG. 10. $x_{s 0}$ is the abscissa of the steady-state and $\rho$ the discount factor. The two lines in (a) cross the graph at one point, but the two lines in (b) cross the graph at three points.

Appendix A. Lemma 2.1, Let us consider (2.7):

$$
\left\{\begin{array}{l}
x^{\prime}(t)=f_{1}(x, a), \\
a^{\prime}(t)=f_{2}(x, a) .
\end{array}\right.
$$

By solving the system

$$
\left\{\begin{array}{l}
f_{1}(x, a)=0 \\
f_{2}(x, a)=0
\end{array}\right.
$$

with respect to $x$, we deduce that

$$
k(x)=1.2 x^{6}-2 x^{5}+(1.8-\rho) x^{4}-2 x^{3}-2 \rho x^{2}+2 x-(0.6+\rho)=0 .
$$

For a given $\rho$, the roots of $k(x)$ in $[0,2]$ are the steady-states of the dynamical system (2.7) in $(0,2]$. We have that $k(0)=-(0.6+\rho)<0$ and $k(2)=29-25 \rho>0$ for $\rho \in(0,0.4]$, so $k(x)$ has 1 or 3 or 5 roots in $[0,2]$. Assuming that there are 5 roots in $[0,2]$, then $k(x)$ should have 6 real roots. Taking its fourth derivative and applying Rolle's Theorem successively four times we deduce that $k^{(4)}(x)=432 x^{2}-240 x+43.2-24 \rho$ has 2 roots in $\mathbb{R}$. Its discriminant is $D=-17049.6+41472 \rho$ and for $\rho \in(0,0.4], D$ is negative, which is a contradiction. We conclude that $k(x)$ has 1 or 3 roots in $[0,2]$, which means that there are 1 or 3 steady-states of (2.7) in $[0,2]$.

Appendix B. Range of the discount factor values for one or three steadystates. We now investigate the range of $\rho$ for the two cases described in Lemma 2.1 to occur. Solving (A.1) with respect to $\rho$ we get

$$
\rho=\frac{1.2 x_{s 0}{ }^{6}-2 x_{s 0}{ }^{5}+1.8 x_{s 0}{ }^{4}-2 x_{s 0}{ }^{3}+2 x_{s 0}-0.6}{\left(x_{s 0}{ }^{2}+1\right)^{2}},
$$

where $x_{s 0}$ is the abscissa of the steady-state. The graph of $\rho-x_{s 0}$ is depicted in Figs. 10(a) and 10(b). The lines $\rho=0.096$ and $\rho=0.164$ cross the graph at one point, but the lines $\rho=0.097$ and $\rho=0.163$ cross the graph at three points.

Rounding $\rho$ to the nearest thousandth, we have that 
- if $\rho \in(0,0.096]$, then there is one steady-state,

- if $\rho \in[0.097,0.163]$, then there are three steady-states,

- if $\rho \in[0.164,0.4]$, then there is one steady-state.

REMARK B.1. If $\rho_{m}, \rho_{M}$ are the local minimum and local maximum respectively of the function

$$
S\left(x_{s 0}\right)=\frac{1.2 x_{s 0}{ }^{6}-2 x_{s 0}{ }^{5}+1.8 x_{s 0}{ }^{4}-2 x_{s 0}{ }^{3}+2 x_{s 0}-0.6}{\left(x_{s 0}{ }^{2}+1\right)^{2}}
$$

in $[0,2]$, then for $\rho \in\left(0, \rho_{m}\right) \cup\left(\rho_{M}, 0.4\right]$ there is one steady-state and for $\rho \in\left(\rho_{m}, \rho_{M}\right)$ there are three steady-states. For $\rho=\rho_{m}$ or $\rho=\rho_{M}$, A.1 has one double and one single root. This is a degenerate case, appearing at the boundary of the two domains of $\rho$ where the two cases occur and it is not considered in this work.

Appendix C. Lemma 2.2. We consider a necessary and sufficient condition for the steady-state $\left(x_{s 0}, a_{s 0}\right)$ to be a saddle point by calculating the stability matrix for system (2.7) at $\left(x_{s 0}, a_{s 0}\right)$ and computing its eigenvalues $\lambda$. We end up with the following quadratic equation:

$$
\lambda^{2}-\rho \lambda+2 a_{s 0} t\left(x_{s 0}\right)=0,
$$

where $t\left(x_{s 0}\right)=\frac{-0.6 x_{s 0}\left(x_{s 0}{ }^{2}+1\right)^{3}+2 x_{s 0}{ }^{2}\left(x_{s 0}{ }^{2}+1\right)+3 x_{s 0}{ }^{2}-1}{\left(x_{s 0}+1\right)^{3}}$. Then, $\left(x_{s 0}, a_{s 0}\right)$ is a saddle point if and only if the quadratic equation has two roots, one positive and one negative, and this is the case if and only if $2 a_{s 0}\left(t\left(x_{s 0}\right)-a_{s 0}\right)<0$. We have that $a_{s 0}>0$, so we finally conclude that our necessary and sufficient saddle-point condition is $t\left(x_{s 0}\right)<a_{s 0}$. Since $a_{s 0}=0.6 x_{s 0}-\frac{x_{s 0}^{2}}{x_{s 0}{ }^{2}+1}$ and by denoting $w\left(x_{s 0}\right)=0.6 x_{s 0}-\frac{x_{s 0}^{2}}{x_{s 0}{ }^{2}+1}$, the above condition is written in the form

$$
w\left(x_{s 0}\right)>t\left(x_{s 0}\right) .
$$

The graphs of $w\left(x_{s 0}\right), t\left(x_{s 0}\right)$ in the $\left(x_{s 0}, a_{s 0}\right)$ diagram are depicted in Fig. 11(a). Rounding to the nearest thousandth, we have that $\left(x_{s 0}, a_{s 0}\right)$ is a saddle point provided that $x_{s 0} \in[0,0.626) \cup(1.063,2]$.

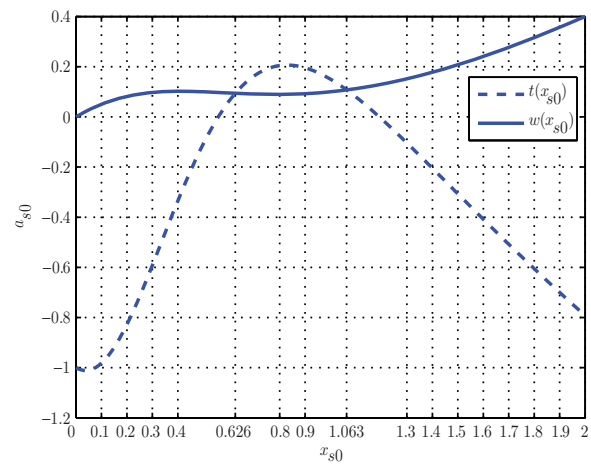

(a)

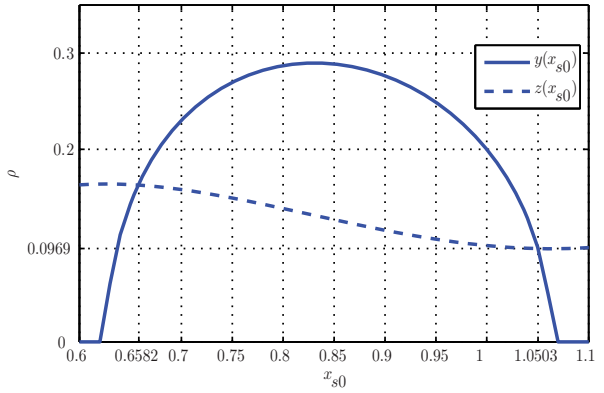

(b)

FIG. 11. Graphs used in the proof of Lemma $2.2 x_{s 0}, a_{s 0}$ are the coordinates of the steady-state and $\rho$ is the discount factor. 
REMARK C.1. If $x_{1}, x_{2}$ are the roots of $w\left(x_{s 0}\right)-t\left(x_{s 0}\right)=0$ in $[0,2], x_{1}<x_{2}$, and $\left(x_{s 0}, a_{s 0}\right)$ is a saddle point provided that $x_{s 0} \in\left[0, x_{1}\right) \cup\left(x_{2}, 2\right]$.

As mentioned in Lemma 2.1, we have the following cases:

- There is one steady state. Then $\rho \leq 0.096$ or $\rho \geq 0.164$. We have that $k(0.626)=$ $0.3177-1.9373 \rho$ and $k(1.063)=0.4388-4.5363 \rho$. We have shown that $k(0)<0$ and $k(2)>0$. If $\rho \leq 0.096$, then $k(0.626)>0$, so $x_{s 0} \in[0,0.626]$ and we conclude that $\left(x_{s 0}, a_{s 0}\right)$ is a saddle point. If $\rho \geq 0.164$, then $k(1.063)<0$, so $x_{s 0} \in(1.063,2]$ and we make the same conclusion. So, $\left(x_{s 0}, a_{s 0}\right)$ is always a saddle point.

- There are three steady-states. Then, $0.097 \leq \rho \leq 0.163$. For $\rho$ in this range, we have that $k(0.626)>0$ and $k(1.063)<0$. So, if $\left(x_{s 1}, a_{1}\right),\left(x_{s 2}, a_{2}\right),\left(x_{s 3}, a_{3}\right)$ are the three points, then $x_{s 1} \in(0,0.626), x_{s 2} \in(0.626,1.063), x_{s 3} \in(1.063,2)$, which means that the first and third points are saddle points but the second one is not. We focus on the second point. We find the discriminant $D$ of the quadratic equation (C.1). Then $\left(x_{s 0}, a_{s 0}\right)$ is a vortex (with outward direction) iff $D<0$ (if $D<0$, then $2 \operatorname{Re}\left(\lambda_{i}\right)=-\frac{-\rho}{1}>0$, so (C.1) has two complex conjugate roots with positive real parts). The previous condition is written in the form

$$
\begin{aligned}
& \rho<\frac{\sqrt{8\left[0.6 x_{s 0}\left(x_{s 0}^{2}+1\right)-x_{s 0}^{2}\right]}}{x_{s 0^{2}+1}} . \\
& . \frac{\sqrt{\left[-1.2 x_{s 0}\left(x_{s 0}{ }^{2}+1\right)^{3}+2 x_{s 0}^{2}\left(x_{s 0}{ }^{2}+1\right)+x_{s 0}{ }^{2}\left(x_{s 0}{ }^{2}+1\right)^{2}+3 x_{s 0}{ }^{2}-1\right]}}{x_{s 0}{ }^{2}+1} .
\end{aligned}
$$

Denoting by $y\left(x_{s 0}\right)$ the right part of this inequality and writing relation (B.1) as $\rho=z\left(x_{s 0}\right)$, we conclude that $\left(x_{s 0}, a_{s 0}\right)$ is a vortex if and only if

$$
y\left(x_{s 0}\right)>z\left(x_{s 0}\right) .
$$

The graphs of $y\left(x_{s 0}\right), z\left(x_{s 0}\right)$ in the $\left(x_{s 0}, \rho\right)$ diagram are depicted in Fig. 11(b). Rounding to the nearest thousandth, we have that $\left(x_{s 0}, a_{s 0}\right)$ is a vortex for $x_{s 0} \in[0.658,1.050]$.

From Fig. 10(b), approximating to the fourth decimal digit, we see that $x_{s 2} \in[0.6551,1.0458]$. So $\left(x_{s 2}, a_{2}\right)$ is a vortex, except for some degeneracies (as is also mentioned in [18, page 538) which are met when $x_{s 2} \in[0.6551,0.658)$ (e.g. if $\rho=0.163$, then $\left(x_{s 2}, a_{2}\right)=(0.6551,0.0928)$ and the quadratic equation (C.1) has two positive real roots, so $\left(x_{s 2}, a_{2}\right)$ is an unstable node).

\section{REFERENCES}

[1] L. Ambrosio, P. Cannarsa, and H. M. Soner, On the propagation of singularities of semi-convex functions, Annali Scuola Norm. Sup. Pisa 20(4) (1993), 597-616. MR1267601 (95b:49068)

[2] M. Bardi and I. Capuzzo-Dolcetta, Optimal control and viscosity solutions of Hamilton-JacobiBellman equations, Birkhäuser, Boston, 1997. MR 1484411(99e:49001)

[3] G. Barles, Solutions de viscosité des équations de Hamilton-Jacobi, Mathématiques \& Applications 17, Springer-Verlag 1994. MR1613876 (2000b:49054)

[4] G. Barles and P. E. Souganidis Convergence of approximation schemes for fully nonlinear second order equations, Asymptotic Anal. 4 (1991), 271-283. MR1115933 (92d:35137)

[5] W. A. Brock and D. Starrett, Managing systems with non-convex positive feedback, Environmental \& Resource Economics 26 (2003) 575-602. 
[6] P. Cannarsa and C. Sinestrari, Semiconcave functions, Hamilton-Jacobi equations, and optimal control, Progress in Nonlinear Differential Equations and Their Applications V. 58, Birkhäuser Boston Inc., Boston, MA, 2004. MR2041617 (2005e:49001)

[7] P. Cannarsa and H. M. Soner, On the singularities of the viscosity solutions to Hamilton-JacobiBellman equations, Indiana Univ. Math. J. 36(3) (1987), 501-524. MR.905608 (89m:35044)

[8] S. R. Carpenter, D. Ludwig and W. A. Brock, Management of eutrophication for lakes subject to potentially irreversible change, Ecological Applications, 9(3) (1999), 751-771.

[9] M. G. Crandall, L. C. Evans and P. L. Lions, Some properties of viscosity solutions of HamiltonJacobi equations, Trans. Amer. Math. Soc. 282 (1984), 487-502. MR732102 (86a:35031)

[10] M. G. Crandall and P. L. Lions, Viscosity Solutions of Hamilton-Jacobi equations, Trans. Amer. Math. Soc. 277 (1983), 1-42. MR690039 (85g:35029)

[11] W. Fleming and R. Rishel, Deterministic and Stochastic Optimal Control, Springer, 1975. MR0454768 (56:13016)

[12] L. Grüne, M. Kato and W. Semmler, Solving ecological management problems using dynamic programming, Journal of Economic Behavior \& Organization, 57 (2005), 448-473.

[13] R. Jensen and P. E. Souganidis, A regularity result for viscosity solutions of Hamilton-Jacobi equations in one space dimension, Trans. Amer. Math. Soc. 301, no. 1 (1987), 137-147. MR879566 (88h:35020)

[14] K. Kawaguchi, Optimal Control of Pollution Accumulation with Long-Run Average Welfare, Environmental and Resource Economics 26(3) (2003), 457-468.

[15] K-G. Mäler, A. Xepapadeas, and A. de Zeeuw, The Economics of Shallow Lakes, Environmental and Resource Economics 26(4) (2003), 603-624.

[16] A. Seierstad and K. Sydsæter, Optimal control theory with economic applications, Advanced Textbooks in Economics V. 24, 1987. MR887536 (88h:49002)

[17] J. A. Sethian, Level Set Methods and Fast Marching Methods (2nd edition), Cambridge University Press, 1999. MR1700751 (2000c:65015)

[18] A. K. Skiba, Optimal growth with a convex-concave production function, Econometrica 46(3) (1978), 527-539. MR 491898(81e:90029)

[19] H. M. Soner, Optimal control with state space constraint I, SIAM J. Control Optim. 24 (1986) 552-562; II, 24 (1986) 1110-1122. MR838056 (87e:49029)

[20] J. Stoer and R. Bulirsch, Introduction to numerical analysis (3rd edition), New York: SpringerVerlag, 2002. MR.1923481 (2003d:65001)

[21] F. O. O. Wagener, Skiba points and heteroclinic bifurcations, with applications to the shallow lake system, J. Econom. Dynam. Control 27, no. 9 (2003), 1533-1561. MR1962514 (2003m:91118) 